\title{
Grundfragen staatlicher Erziehungsambitionen
}

\author{
Stefan Huster
}

I. Staatliche Erziehungsambitionen in der Diskussion

II. Die öffentliche Schule als Kernbereich der Erziehungsproblematik

1. Das Spannungsverhältnis von Neutralitätsgebot und Erziehungsauftrag

2. Recht und Pädagogik

3. Das Gleichordnungs- und Abwägungsmodell der Verfassungsrechtsprechung

4. Probleme des Modells

5. Das Abschichtungsmodell als Alternative

6. Folgerungen für die Erziehung in der öffentlichen Schule

7. Schulische „Werteerziehung“ im Lichte des Neutralitätsgebots

a) Ethikunterricht in der öffentlichen Schule

b) Religionsunterricht in der öffentlichen Schule

c) Das Verhältnis von Religions- und Ethikunterricht

III. Staatliche Gesundheitserziehung zwischen Paternalismus und Autonomieförderung

1. Auf dem Weg zur Gesundheitserziehungsdiktatur?

2. Begründungen einer verhaltensbezogenen Public-Health-Politik

3. Die Public-Health-Perspektive

4. Juristische Konsequenzen

IV. Fazit

\section{Staatliche Erziehungsambitionen in der Diskussion}

In der staatstheoretischen und sozialphilosophischen Diskussion wird - etwa in dem sogenannten BöcKENFÖRDE-Paradox ${ }^{1}$ - häufig betont, dass Staat und Recht in vielfältiger Weise auf entgegenkommende Überzeugungen und Lebensformen der Bürger ${ }^{2}$ angewiesen seien. Diese Überzeugungen und Lebensformen sind gelegentlich sogar zu Staats- und Verfassungsvoraussetzungen oder -erwartungen erklärt worden, wobei nicht immer deutlich wird, ob diese Begriffe

1 Ernst-Wolfgang Böckenförde, Die Entstehung des Staates als Vorgang der Säkularisation, in: ders., Recht, Staat, Freiheit, 2. Aufl. 1992, S. 92, 112.

2 Zum Begriff der ,entgegenkommenden Lebensformen“ vgl. Jürgen Habermas, Moralität und Sittlichkeit, in: Wolfgang Kuhlmann (Hrsg.), Moralität und Sittlichkeit, 1986, S. $16,28$. 
verfassungstheoretische oder verfassungsrechtliche Bedeutung besitzen sollen. ${ }^{3}$ Jedenfalls liegt auf der Grundlage dieser Prämisse der Schluss nahe, dass es dem Staat und dem staatlichen Recht auch ein Anliegen sein müsse, bestimmte Überzeugungen und Haltungen hervorzubringen, zu fördern und zu pflegen - und in diesem Sinne die Bürger gegebenenfalls auch zu ,erziehen“.

Gleichzeitig bestehen aber erhebliche Aversionen gegen staatliche Erziehungsambitionen. Diese kann man getrost zur Seite legen, soweit sie lediglich instrumentalisiert werden, um die eigenen Erziehungsvorstellungen durchzusetzen. Tatsächlich findet man dies auch und gerade in der verfassungsrechtlichen Literatur nicht selten: Kritisiert wird der Erziehungsgedanke als solcher, gemeint sind aber spezifische Erziehungsziele und -inhalte (etwa die Erziehung zur Kritikfähigkeit in der öffentlichen Schule). Dies wird immer dann offensichtlich, wenn von denselben Autoren an anderer Stelle eine staatliche Erziehung anderen Inhalts (etwa zu einer entschieden affirmativen Haltung zur bestehenden Rechts- und Gesellschaftsordnung) befürwortet oder sogar eingefordert wird. ${ }^{4}$ Wendet man sich den ernsthafteren Einwänden zu, die grundlegende Konsistenzanforderungen erfüllen, so lassen sich diese anhand folgender Stichworte in zwei Gruppen unterteilen: Form und Inhalt.

Formbezogene Einwände gehen von der Prämisse aus, dass für den freiheitlichen Staat die Unterscheidung von äußerem Verhalten und inneren Motiven seiner Bürger konstitutiv ist: Will er nicht totalitär werden, so muss dem Staat der Zugriff auf die Gesinnungen der Bürger grundsätzlich verwehrt sein; er gibt sich damit zufrieden, dass die Verhaltensanweisungen des staatlichen Rechts befolgt werden - unabhängig davon, ob diesem regelkonformen Verhalten eine inhaltliche Akzeptanz der rechtlichen und politischen Entscheidungen oder lediglich die Absicht zugrunde liegt, die Reaktionen des staatlichen Sanktionsapparats zu vermeiden. Für dieses „Moment der Äußerlichkeit“ ${ }^{“ 5}$ des Staates und des staatlichen Rechts wird gern die - allerdings auf einen spezifischen Zusammenhang zugeschnittene - Aussage des Bundesverfassungsgerichts herangezogen, der Staat habe „nicht die Aufgabe, seine Bürger zu ,bessern'“. ${ }^{6}$ Diese in der kantischen

3 Zur Diskussion vgl. Christoph Möllers, Staat als Argument, 2000, S. 257 ff.; Stefan Huster, Die ethische Neutralität des Staates, 2002, S. 647 ff.

$4 \mathrm{Zu}$ Beispielen für diese inkonsistente und interessengeleitete Argumentation vgl. Huster (Anm. 3), S. 314 f. Fn. 270.

5 Ernst-Wolfgang Böckenförde, Der Staat als sittlicher Staat, 1978, S. 25.

$6 \mathrm{Zu}$ dem vollständigen Zitat, aus dem gleichzeitig der spezifische Kontext deutlich wird, vgl. BVerfGE 22, S. 180, 219 f.: „Der Staat hat aber nicht die Aufgabe, seine Bürger zu ,bessern' und deshalb auch nicht das Recht, ihnen die Freiheit zu entziehen, nur um sie zu ,bessern', ohne dass sie sich selbst oder andere gefährden, wenn sie in Freiheit blieben." 
Tradition der Unterscheidung von Legalität und Moralität ${ }^{7}$ stehende Auffassung bezieht sich umfassend auf das Verhältnis des einzelnen zum Staat und zur Rechtsordnung: Der „Grundrechtsstaat darf nur äußeres Verhalten erzwingen, nicht aber die Moralität seiner Rechtssubjekte. “8 Nimmt man diese Auffassung beim Wort, so ist jede Form der staatlichen Erziehung schon deshalb bedenklich, weil Erziehung per definitionem die Anschauungen und die Persönlichkeitsstruktur des Educanden zu beeinflussen versucht. ${ }^{9}$ Und wenn diese ,inneren" Gegenstände dem staatlichen Zugriff von vornherein und absolut entzogen sind, so kann es auch keine Rolle spielen, welche Erziehungsziele verfolgt und welche Inhalte vermittelt werden.

Nun wird diese Position in dieser Radikalität kaum vertreten, und sie wäre auch mit dem geltenden Recht kaum vereinbar: Denn sowohl das Grundgesetz als auch die Schulgesetze der Länder gehen jedenfalls für einen Lebensbereich ersichtlich von einer staatlichen Erziehungskompetenz aus: nämlich für die öffentliche Schule. Anerkennt man diese Kompetenz, so mögen sich immer noch formbezogene Unterscheidungen - etwa zwischen Erziehung und Indoktrination $^{10}$ - treffen lassen, aber die Diskussion verlagert sich dann auf einen inhaltlichen Gesichtspunkt: Welche Erziehungsziele darf der Staat verfolgen?

\section{Die öffentliche Schule als Kernbereich der Erziehungsproblematik}

\section{Das Spannungsverhältnis von Neutralitätsgebot und Erziehungsauftrag}

Unter diesem inhaltlichen Gesichtspunkt ergibt sich die grundlegende Herausforderung aus dem Gebot der staatlichen Neutralität: ${ }^{11}$ Der freiheitliche Verfassungsstaat zeichnet sich dadurch aus, dass er es den Bürgern überlässt, wie sie ihr

7 Vgl. Immanuel Kant, Die Metaphysik der Sitten, in: ders., Werke in zehn Bänden, hrsg. von Wilhelm Weischedel, Bd. 7, 1983, S. $303 \mathrm{ff}$.

8 Philip Kunig, Rechtsfragen ethischer und religiöser Erziehung in der Schule, in: Gottfried Adam/Friedrich Schweitzer (Hrsg.), Ethisch erziehen in der Schule, 1996, S. 301, 303.

9 Auf diese Besonderheit der Erziehung gegenüber anderen staatlichen Maßnahmen weist auch Ekkehart Stein, Das Recht des Kindes auf Selbstentfaltung in der Schule. Verfassungsrechtliche Überlegungen zur freiheitlichen Ordnung des Schulwesens, 1967, S. 3 hin.

10 Vgl. näher dazu unten II. 4.

11 Zum Folgenden vgl. ausführlich Huster (Anm. 3), S. 272 ff.; Ders., Staatliche Neutralität und schulische Erziehung. Einige Anmerkungen aus verfassungsrechtlicher und so- 
Leben gestalten - worin sie also etwa ihren Lebenssinn finden, welche religiösen und weltanschaulichen Überzeugungen sie besitzen und in welchen Formen sie mit anderen Menschen zusammenleben. Gegenüber diesen Konzeptionen des guten Lebens - oder kürzer: Konzeptionen des Guten oder ethischen Einstellungen und Überzeugungen - verhält sich der Staat grundsätzlich neutral. Seine Regelungsbefugnis beschränkt sich in einer pluralistischen Gesellschaft, in der es unter den Bürgern permanent und auf begründete Weise umstritten ist, wie man leben soll, auf die gerechte Ordnung des Zusammenlebens. Nach dieser Unterscheidung der Fragen des Guten und des Gerechten darf der Staat nur insoweit tätig werden, als seine Maßnahmen auf Gründen beruhen, die unabhängig von einer partikularen ethischen Einstellung nachvollzogen werden können. Machte er sich dagegen eine der umstrittenen Überzeugungen zu eigen, so behandelte er nicht alle Bürger mit gleicher Achtung und gleichem Respekt; er wäre dann nicht mehr - wie es das Bundesverfassungsgericht geradezu anrührend formuliert hat - „Heimstatt aller Bürger“. ${ }^{12}$

Dabei ist es für den Bereich der öffentlichen Schule eine besondere Herausforderung, den Gehalt des Neutralitätsgebots zu explizieren. Auf der einen Seite nimmt der Staat als Träger der öffentlichen Schule einen eigenständigen Erziehungsauftrag für sich in Anspruch, der in Art. 7 Abs. 1 GG eine - wenn auch nicht sehr deutliche - verfassungsrechtliche Verankerung findet. ${ }^{13}$ Dementsprechend enthalten die Verfassungen und Schulgesetze der Bundesländer, die in schulrechtlichen Fragen zuständig sind, auch umfangreiche und gelegentlich recht ambitionierte Kataloge von Erziehungszielen. Diese reichen von politischen Tugenden im engeren Sinne (,Erziehung zu freiheitlicher demokratischer Gesinnung") über sozialmoralische Vorgaben („Erziehung zu sittlicher Verantwortlichkeit“) bis hin zu Leitlinien der Gestaltung des eigenen Lebens (,Aufgeschlossenheit für alles Wahre, Gute und Schöne“, aber auch „Ehrfurcht vor Gott"). In einer Offenheit, die aus anderen Rechtsbereichen nicht bekannt ist, maßt sich die öffentliche Gewalt somit an, in der Schule Einfluss auf die Persönlichkeitsentwicklung der Bürger zu nehmen. Insbesondere in dem Moment, in dem der Schule wieder zunehmend die Aufgabe einer "Werteerziehung“" zugeschrieben wird, stellt sich die Frage, wie und mit welchem Ziel dies geschehen soll und darf. Die politischen und juristischen Debatten um bayerische Schulkreuze, sächsische und nordrhein-westfälische „Kopfnoten“, brandenbur-

zialphilosophischer Sicht, Neue Sammlung. Vierteljahres-Zeitschrift für Erziehung und Gesellschaft 41 (2001), S. 399 ff.

12 BVerfGE 19, S. 206, 216.

13 Vgl. dazu zuletzt Matthias Jestaedt, Schule und außerschulische Erziehung, in: Josef Isensee/Paul Kirchhof (Hrsg.), Handbuch des Staatsrechts der Bundesrepublik Deutschland, Bd. 7, 3. Aufl. 2009, \$ 156, Rn. 38 ff. 
gische Lebenskunde sowie den Religions- und Ethikunterricht in Berlin und Baden-Württemberg indizieren, dass die „Werteerziehung“ wieder auf der Tagesordnung steht.

Der Bildungs- und Erziehungsauftrag der öffentlichen Schule findet seine sachliche Legitimation in der Persönlichkeitsentwicklung des Schülers, daneben aber auch in dem Bemühen, zur - wie es in der verfassungsrechtlichen Literatur reichlich unscharf, aber wohl kaum völlig unzutreffend häufig formuliert wird Integration des Gemeinwesens beizutragen. Es ist daher nicht überraschend, dass die Schule in besonderer Weise von den „Grundwerte“-Diskussionen erfasst wird, die in regelmäßigen Abständen das Land überziehen. ${ }^{14}$ Auf der anderen Seite läuft aber gerade eine öffentliche Pflichtschule, die entschiedene Erziehungsambitionen hegt, immer Gefahr, mit den verfassungsrechtlich garantierten Grundrechten der Schüler und ihrer Eltern zu kollidieren, sich nicht staatlicherseits vorschreiben lassen zu müssen, welche Überzeugungen, Lebensformen und - dieser äußerst unglückliche Begriff lässt sich nur schwer vermeiden - „Werte" vorzugswürdig sind; zumal in einer pluralistischen oder gar multikulturellen Gesellschaft insoweit durchaus unterschiedliche Vorstellungen bestehen werden. Das Dilemma besteht also darin, dass die öffentliche Schule einerseits einen Erziehungsauftrag besitzt, der zudem zunehmend betont wird, andererseits aber als staatliche Einrichtung mit Pflichtcharakter nicht die grundrechtliche Freiheit der Bürger einschränken darf, selbst über die Gestaltung ihres Lebens zu entscheiden. Anders formuliert: Wie ist eine schulische Erziehung möglich, die das verfassungsrechtliche Neutralitätsgebot beachtet?

\section{Recht und Pädagogik}

Das beschriebene Dilemma ist hier als ein Problem des Rechts, insbesondere des Verfassungsrechts formuliert worden. Darin liegt bereits eine gewisse Abstraktion, denn in der schulischen Lebenswelt handelt es sich natürlich zuallererst um ein Thema der praktischen Erziehung und ihrer Wissenschaft, der Pädagogik. Dies könnte zu der Ansicht verleiten, dass das Recht und die Juristen hier gar nichts zu suchen haben: Bildungs- und Erziehungsprozesse folgen einer eigenen Logik, die von Rechtsnormen im besten Fall unberührt bleibt, im schlimmsten Fall zerstört wird.

Die berechtigten Hinweise auf die Grenzen der Verrechtlichung dieses Lebensbereichs können aber nicht darüber hinwegtäuschen, dass man das Recht insoweit nicht völlig außen vor lassen kann. Die Ausgestaltung der schulischen

14 Bereits ein Locus classicus der Vorgängerdebatte: GüNTER GorscheneK (Hrsg.), Grundwerte in Staat und Gesellschaft, 1977. 
Bildung und Erziehung stellt für das Selbstverständnis eines Gemeinwesens ein zentrales Thema dar, das auch politisch in dieser Weise wahrgenommen wird. Es kann daher nicht einer „pädagogischen Autonomie“ der Schule und der Lehrer überlassen werden, sondern muss zumindest in seinen Grundsätzen - wie die Verfassungsrechtsprechung zu Recht betont hat - durch den Gesetzgeber geregelt werden. ${ }^{15}$ Bei allen Problemen, die damit aus pädagogischer Sicht verbunden sein mögen, ist es ein enormer rechtsstaatlicher Fortschritt, dass die Schule nicht mehr als ein rechtsfreier Raum betrachtet wird. Gleichzeitig muss der Lebenssachverhalt „Schule“ nicht nur rechtlich geformt werden, sondern dieses Schulrecht unterliegt seinerseits wiederum verfassungsrechtlichen Grenzen: Auch und gerade im grundrechtssensiblen Bereich der schulischen Einwirkung auf die Persönlichkeitsentwicklung muss sich das staatliche Handeln an den verfassungsrechtlichen Vorgaben messen lassen.

Dies heißt nicht, dass jedes schulische Detail rechtlich geregelt werden muss; nicht jede Curricularnorm ist verfassungsrechtlich geboten. Auch ist es selbstverständlich möglich zu berücksichtigen, dass die Schule keine typische Verwaltungsbehörde darstellt, sondern hier Bildungs- und Erziehungsprozesse stattfinden, die ihren Eigensinn besitzen. Aber dies sind Argumente, die sich innerhalb des Rechtssystems zur Geltung bringen müssen, um schulische Freiräume zu begründen; sie rechtfertigen keinen Totalvorbehalt gegenüber gesetzlichen Vorgaben und rechtlicher Kontrolle.

\section{Das Gleichordnungs- und Abwägungsmodell der Verfassungsrechtsprechung}

Auch die erzieherische Einwirkung in der Schule unterliegt demnach der verfassungsrechtlichen Kontrolle. Das Bundesverfassungsgericht hat diese Einsicht umgesetzt, indem es gegen den schulischen Erziehungsauftrag (Art. 7 Abs. 1 GG) die Grundrechte der Schüler und vor allem das elterliche Erziehungsrecht (Art. 6 Abs. 2 GG) in Stellung gebracht hat. Damit ist zunächst geklärt, dass die Grundrechte nicht vor der Schultüre enden: Die Eltern können sich grundsätzlich unter Berufung auf ihr Erziehungsrecht gegen die schulische Erziehung wenden. Schulischer Erziehungsauftrag und elterliches Erziehungsrecht seien nicht strikt separiert, sondern in der Schule ,gleichgeordnet“. Aus dieser Gleichordnung folge, dass Eltern und Schule eine ,gemeinsame Erziehungsaufgabe“ besitzen, die in einem ,sinnvoll aufeinander bezogenen Zusammenwirken " zu erfüllen sei. Ausschlaggebend für diese Position des Verfassungsgerichts ist - neben entstehungsgeschichtlichen und verfassungssystematischen Argu-

15 Zum Gesetzesvorbehalt im Schulrecht vgl. grundlegend BVerfGE 47, S. 46 ff. 
menten - die Annahme, dass sich elterliche und schulische Erziehung auf die „Bildung der einen Persönlichkeit des Kindes“ beziehen und sich daher ,nicht in einzelne Komponenten zerlegen " lassen. ${ }^{16}$

Allerdings stellt sich dann die Frage, wie die Erziehungskompetenzen in der öffentlichen Schule abzugrenzen sind, umso schärfer. Die Rechtsprechung antwortet darauf mit einer Reihe von Differenzierungen und Ausgleichsvorgaben. Zunächst liege die Verantwortung für den „Gesamtplan“ der Erziehung bei den Eltern. Für die öffentliche Schule folge daraus ein Gebot der Zurückhaltung: Sie dürfe sich ,nicht anmaßen [...], die Kinder in allem und jedem zu unterrichten“, und müsse „für die Vielfalt der Anschauungen in Erziehungsfragen soweit offen sein, als es sich mit einem geordneten staatlichen Schulsystem verträgt." Ferner liegt der Rechtsprechung eine Unterscheidung verschiedener Erziehungsbereiche zugrunde. So komme z.B. der Sexualerziehung, grundsätzlich eine größere Affinität zum elterlichen Bereich als zum schulischen Sektor“ zu. Gleiches gilt in der Sache auch für die religiös-weltanschauliche Erziehung. Demnach müssten aber auch andere Bildungs- und Erziehungsinhalte denkbar sein, die eher in den schulischen Bereich fallen. Dies soll - wohl weithin unabhängig von ihrem Inhalt - für die ,,bloße Wissensvermittlung“ gelten, die ,typischerweise“ Aufgabe der insoweit auch besser als das Elternhaus geeigneten Schule sei.

Diese Abgrenzungsversuche vermögen einen Konflikt von schulischen und elterlichen Erziehungsvorstellungen aber nicht von vornherein auszuschließen, weil sie beide Erziehungssphären gerade nicht trennscharf voneinander scheiden. Es sind daher schließlich Abwägungs- und Kompromissregeln erforderlich, die ein „sinnvoll aufeinander bezogenes Zusammenwirken“ ermöglichen. Dazu gehören - insbesondere in den Erziehungsfragen, die primär dem häuslichen Bereich angehören sollen - Informationsansprüche der Eltern, damit die Erziehung in ,größtmöglicher Abstimmung zwischen Eltern und Schule geplant und durchgeführt werden“ kann und die Eltern in der Lage sind, „das ihnen nach dem Grundgesetz vorrangig zustehende individuelle Erziehungsrecht zur Geltung zu bringen." Auch das Gebot, in sensiblen Bereichen die ,gebotene Zurückhaltung und Toleranz" in der schulischen Erziehung zu beachten und den Versuch einer „Indoktrinierung der Schüler“ zu unterlassen, gehört zu diesen Konkordanzformeln.

16 Vgl. dazu und zum folgenden Text insbesondere die Entscheidung BVerfGE 47, S. 46 ff. zum Sexualkundeunterricht. Vgl. ferner Jestaedt (Anm. 13), \$ 156, Rn. 83 ff.; HansUlrich Evers, Die Befugnis des Staates zur Festlegung von Erziehungszielen in der pluralistischen Gesellschaft, 1979; und die Beiträge von Michael Bothe und Armin DitTmann, Erziehungsauftrag und Erziehungsmaßstab der Schule im freiheitlichen Verfassungsstaat, Veröffentlichungen der Vereinigung der Deutschen Staatsrechtslehrer 54 (1995), S. 7 ff. und S. 47 ff. 


\section{Probleme des Modells}

Diese Theorie der Gleichordnung von staatlichem und elterlichem Erziehungsauftrag in der öffentlichen Schule ist nicht nur deshalb problematisch, weil sie unweigerlich Koordinierungs- und Abwägungsgebote mit sich bringt, die weder der Schule noch den Eltern mit hinreichender Deutlichkeit Maßstäbe an die Hand geben, welche Erziehung in der Schule geboten und zulässig ist. Darüber hinaus führt sie - zumindest in der Fassung, die sie in der Rechtsprechung des Bundesverfassungsgerichts gewonnen hat - zu einer Bestimmung des Verhältnisses der konkurrierenden Erziehungsbefugnisse, die schwerlich überzeugen kann.

Wenn man davon ausgeht, dass sich elterliche und schulische Erziehungsbemühungen auf die „Bildung der einen Persönlichkeit des Kindes“ beziehen und sich die Erziehung daher „nicht in einzelne Komponenten zerlegen“ lässt, so ist es konsequent, jede Form der erzieherischen Einwirkung in der öffentlichen Schule zumindest dann als Eingriff in das Elternrecht zu qualifizieren, wenn diese Einwirkung den elterlichen Vorstellungen widerspricht. Eine den elterlichen Vorstellungen widersprechende schulische Erziehung wäre damit nicht schlechthin unzulässig; wohl aber müsste die Schule auf die Vorstellungen der Eltern - insbesondere auf ihren „Gesamterziehungsplan“ - jeweils Rücksicht nehmen und ihre eigenen Ambitionen mit den elterlichen Vorstellungen abwägen. Diese Konstruktion führt nicht notwendigerweise zu unplausiblen Ergebnissen; aus dieser Abwägung mag sich nämlich im Einzelfall ergeben, dass die elterlichen Vorstellungen vollständig zurücktreten müssen. Der sachlichen Problematik unangemessen ist aber der dogmatische Grundansatz: ${ }^{17}$ Dass die elterlichen Erziehungsvorstellungen grundsätzlich immer in Rechnung zu stellen sind, verträgt sich nur schwer mit der weithin geteilten Ansicht, dass in der öffentlichen Schule bestimmte Inhalte und auch normative Vorgaben ganz unabhängig davon vermittelt werden dürfen, ob sie mit den Vorstellungen der betroffenen Eltern übereinstimmen. Ob und in welchem Umfang den Schülern unverzichtbare Kenntnisse und Fähigkeiten und grundlegende normative Orientierungen in der Schule nahe gebracht werden, kann und darf nicht davon abhängen, dass die Eltern dagegen keine Einwände erheben. Wenn sich die Eltern beispielsweise mit der Begründung gegen den allgemeinen Geschichtsunterricht wenden, dass hier auch eine Beschäftigung mit der Gedankenwelt und den Institutionen des Christentums stattfinde, die sie ablehnen, so ist dieser Einwand unbeachtlich; keineswegs ist die Schule deshalb verpflichtet, die jeweiligen Schüler vom Geschichtsunterricht zu befreien oder diesen Unterricht zu modifizieren oder

17 Vgl. dazu auch den Diskussionsbeitrag von Stefan Huster, Veröffentlichungen der Vereinigung der Deutschen Staatsrechtslehrer 65 (2006), S. 497 zu Michael Germann, a.a.O., S. $491 \mathrm{f}$. 
gar zu unterlassen. Auch religiös-weltanschauliche Vorbehalte gegen die Evolutionstheorie zwingen die Schule keineswegs dazu, deren Vermittlung zu unterlassen oder ergänzend auch den „Kreationismus“ oder ähnlichen Aberglauben (,Intelligent Design“ u.ä.) darzustellen. Dass dabei keine Abwägung stattfindet, ist schon daran ersichtlich, dass dieses Ergebnis unabhängig davon ist, wie viele Eltern diesen Einwand erheben und in welchem Maße ihre Erziehungsvorstellungen - und möglicherweise sogar ihr "Gesamterziehungsplan“ - durch den Geschichts- oder Biologieunterricht „unterlaufen“ werden. Gleiches gilt für die Vermittlung zentraler politischer Prämissen unseres Gemeinwesens: Dass in der Schule zur Achtung der Menschenwürde, zur Toleranz und zur Unterstützung von Demokratie und Rechtsstaatlichkeit erzogen werden darf, bleibt auch dann richtig, wenn viele Eltern diesen Vorgaben gleichgültig oder ablehnend gegenüberstehen. Fasst man diesen Einwand zusammen, so könnte man sagen, dass das Elternrecht zu weit - und der staatliche Erziehungsauftrag entsprechend zu eng - gefasst ist, wenn alle Erziehungsziele der öffentlichen Schule unter den Vorbehalt gestellt werden, dass sie mit den jeweiligen Erziehungsvorstellungen der Eltern übereinstimmen.

Eine andere Figur, die bei der Bestimmung der Grenzen des staatlichen Erziehungsauftrags eine weite Verbreitung gefunden hat und auch in der Rechtsprechung des Bundesverfassungsgerichts herangezogen wird, ist das Verbot der Indoktrination. Dieses Verbot zielt - zumindest auf den ersten Blick - eher auf die Art und Weise als auf die inhaltliche Tendenz der schulischen Erziehungstätigkeit ab. Seinen Zusammenhang mit dem Elternrecht könnte man in folgender Weise rekonstruieren: Wenn die Schule auf die Erziehungsvorstellungen der Eltern Rücksicht nehmen muss, so ist es nicht zulässig, bestimmte Erkenntnisse und Normen, die die Eltern nicht teilen, den Schülern in einer Form zu vermitteln, die gegenläufige Bildungs- und Erziehungsbemühungen der Eltern leerlaufen lässt. Das Indoktrinationsverbot scheint also eine „zu intensive“ Einwirkung auf die Schüler zu untersagen; und wenn das dadurch geschützte elterliche Erziehungsrecht in der Schule umfassend zur Geltung kommt, ist es nur konsequent, dass auch das Indoktrinationsverbot die öffentliche Schule durchgängig verpflichtet. Fragt man allerdings genauer, was der Begriff der Indoktrination bedeutet, so wird die Lage unübersichtlich. Das Bundesverfassungsgericht hat in der Sexualkunde-Entscheidung formuliert, die Schule müsse „den Versuch einer Indoktrinierung der Schüler mit dem Ziel unterlassen, ein bestimmtes Sexualverhalten zu befürworten oder abzulehnen. "“18 Diese Formulierung kann auf der einen Seite lediglich bedeuten: Es kommt der Schule nicht zu, sich in der Frage des richtigen Sexualverhaltens eine bestimmte Position zu eigen zu

18 BVerfGE 47, S. 46, 77. 
machen und die Schüler zugunsten dieser Position - und zu Ungunsten anderer Ansichten - zu beeinflussen. Tatsächlich lässt sich das Indoktrinationsverbot oft als ein derartiges Verbot der einseitigen Beeinflussung reformulieren. Ein derartiges Beeinflussungsverbot mag in vielen Fällen sinnvoll sein, es kann für die Erziehung in der öffentlichen Schule aber keine umfassende Geltung beanspruchen: Denn zumindest in einigen Erziehungsbereichen ist die Schule sehr wohl berechtigt, bestimmte Normen und Verhaltensweisen als vorzugswürdig darzustellen und zu vermitteln, also mit dem Ziel auf die Schüler einzuwirken, „ein bestimmtes Verhalten zu befürworten oder abzulehnen“. Wenn die Schule einen Erziehungsauftrag besitzt und Erziehung darin besteht, auf der Grundlage von Persönlichkeitsidealen als Erziehungszielen auf die Entwicklung der Kinder und Heranwachsenden - wie moderat auch immer - Einfluss zu nehmen, so ist auch die schulische Erziehung - weil an bestimmten Zielen orientiert, die andere Ziele ausschließen - notwendigerweise einseitig und stellt daher eine Form der Indoktrination in diesem schwachen Sinne dar. Auf der anderen Seite gibt es Formen der suggestiven Beeinflussung, die dem Adressaten keinen eigenen Reflexions- und Entscheidungsspielraum belassen und die deshalb häufig als Indoktrination bezeichnet werden. Hierzu zählen die Methoden der „Umerziehung“ - bis hin zur Gehirnwäsche -, die aus totalitären Staaten bekannt sind; in weniger extremen - und für die schulische Erziehung gewiss einschlägigeren - Fällen mag damit allgemein die aggressive, missionarische, kämpferische, eben „zu intensive“ Einwirkung auf die Schüler gemeint sein. Auch dieser engere Begriff der Indoktrination hilft aber aus zwei Gründen nicht weiter. Zum einen ist es dem freiheitlichen Rechtsstaat in der öffentlichen Schule - und nicht nur dort - zweifellos untersagt, seine Bürger in diesem Sinne zu überwältigen und an der Gewinnung eines eigenen Urteils zu hindern. Nur ergibt sich dieses Verbot schon aus dem Persönlichkeitsrecht des betroffenen Schülers; mit dem Schutz des elterlichen Erziehungsrechts hat es nur mittelbar zu tun. Zum anderen reicht ein in diesem Sinne verstandenes Indoktrinationsverbot nicht aus: Es erlaubt nämlich eine schulische Erziehung, die den Schüler zwar nicht überwältigt - so dass man nicht von Indoktrination in diesem Sinne sprechen kann -, aber doch dezidiert mit einer bestimmten Tendenz beeinflusst. Dies kann nun nicht in allen Bereichen der Schulerziehung zulässig sein: Eine Schule, die gegen den Willen der Eltern für oder gegen eine bestimmte Religion oder eine bestimmte parteipolitische Anschauung erzieht, wird nicht dadurch verfassungsmäßig, dass sie auf Methoden verzichtet, die eine eigene Urteilsbildung schlechthin verhindern. Reduziert man das elterliche Erziehungsrecht auf ein derartiges Indoktrinationsverbot, so ist es zu schwach: Denn in bestimmten Fragen müssen es sich die Eltern überhaupt nicht gefallen lassen, dass die Schule auf ihre Kinder mit einer 
bestimmten Tendenz einwirkt. ${ }^{19}$ In diesem Sinne kann das Verbot der Indoktrination das Neutralitätsprinzip weder konkretisieren noch ersetzen, sondern nur eine zusätzliche Schranke staatlichen Handelns darstellen. Die dargestellten Unklarheiten sind dem Begriff der Indoktrination aufgrund seines normativen und rhetorischen Gehalts immanent: Indoktrination ist etwas Schlechtes, und so bezeichnet man genau diejenige Erziehung als Indoktrination, deren inhaltliche Ausrichtung man ablehnt. In pädagogischer Hinsicht mag man über den Begriff der Indoktrination und seinen Unterschied zum Erziehungsbegriff lange streiten - in der verfassungsrechtlichen Diskussion trägt er nicht sonderlich viel zur Problembewältigung bei. ${ }^{20}$

Die bisherige Diskussion legt das Fazit nahe, dass das Modell der Gleichordnung von staatlichem Erziehungsauftrag und elterlichem Erziehungsrecht in der öffentlichen Schule mit prinzipiellen Schwächen behaftet ist: Entweder das Elternrecht geht zu weit, weil es sich auch gegen obligatorische Erziehungsaktivitäten der Schule wenden kann, oder das Elternrecht wird auf ein unspezifisches Indoktrinationsverbot verengt, das gegen eine in der Form moderate, aber trotzdem ersichtlich einseitige Beeinflussung auf politischem und religiösweltanschaulichem Gebiet keinen Schutz bietet. Dieses Dilemma findet seinen Grund in der Vernachlässigung der inhaltlichen Dimension des staatlichen Erziehungsauftrags: Letztlich muss es für die Schutzwirkung des Elternrechts - und für die verfassungsrechtliche Beurteilung insgesamt - ausschlaggebend sein, wie und wozu die staatliche Schule erziehen darf. Das Gleichordnungsmodell gibt auf diese Frage schon deshalb keine Antwort, weil die Wirkungsrichtung des Elternrechts in diesem Modell davon abhängt, welche Erziehungsziele der einzelne Grundrechtsträger befürwortet oder ablehnt. Angesichts der Pluralität der elterlichen Erziehungsvorstellungen kann sich daraus aber keine nähere Bestimmung des staatlichen Erziehungsauftrags ergeben. Wenn das Gleichordnungsmodell im Einzelfall zu vernünftigen Ergebnissen führen mag, so liegt das nicht daran, dass es der sachlichen Problematik angemessen ist, sondern ist darauf zurückzuführen, dass es aufgrund seiner Vagheit und Unbestimmtheit weithin ergebnisoffen ist.

\section{Das Abschichtungsmodell als Alternative}

Die öffentliche Schule besitzt einen eigenständigen Erziehungsauftrag, dessen nähere Ausgestaltung sich nicht aus verfassungsrechtlichen Vorgaben ergibt. Es bedarf daher einer genuin politischen Entscheidung, welche Bildungsinhalte die staatliche Schule vermitteln und welche Erziehungsziele sie verfolgen soll. Die

19 Dies ist der richtige Sinn insbesondere der Schulkreuz-Entscheidung BVerfGE 93, S. 1 ff.

20 Ähnlich kritisch Jestaedt (Anm. 13), \ 156, Rn. 70. 
Grundrechte und weitere grundgesetzliche Normen determinieren diese Entscheidung nicht, sondern können insoweit dem staatlichen Handeln - wie in anderen Rechtsbereichen auch - lediglich Grenzen setzen. Diese Grenzen können allerdings nicht in einem Verbot der Indoktrination gefunden werden; und sie können sich in einer pluralistischen Gesellschaft auch nicht aus den subjektiven Erziehungsvorstellungen der Eltern ergeben, sondern müssen einen davon unabhängigen, objektiven Charakter besitzen.

Es ist daher an der Grundfrage anzusetzen: Welche Erziehungsbemühungen der staatlichen Schule müssen sich die Schüler und ihre Eltern gefallen lassen? Auf der einen Seite besitzt der Staat einen eigenständigen Erziehungsauftrag; auf der anderen Seite ermächtigt ihn dieser nicht zu beliebigem Erziehungsverhalten. Aus der Sicht der Betroffenen kann nur diejenige Erziehung in der öffentlichen Schule zumutbar sein, für die der staatliche Schulträger prinzipiell einsehbare Gründe vorbringen kann. An genau dieser Stelle setzt das - recht verstandene Prinzip der Neutralität an: Gerechtfertigt ist die schulische Erziehung, soweit sie sich auf neutrale Weise rechtfertigen lässt. Damit ist der staatliche Erziehungsauftrag nicht von vornherein auf bestimmte, inhaltlich „neutrale“ Ziele beschränkt; es kommt für die verfassungsrechtliche Beurteilung vielmehr darauf an, welche Gründe jeweils für eine Erziehungsmaßnahme geltend gemacht werden können. Grundsätzlich zulässig sind demnach Erziehungsziele und -maßnahmen, deren Begründung nicht darauf beruht, dass man in umstrittenen politischen, religiös-weltanschaulichen oder ethischen Fragen eine bestimmte Position vertritt; unzulässig sind dagegen diejenigen Erziehungsziele, deren Plausibilität eben dies voraussetzt.

Von entscheidender Bedeutung ist dabei, dass es mit dem Abstellen auf die Neutralität der Begründung nicht mehr erforderlich ist, dass die staatlichen Erziehungsbemühungen wirkungsneutral sind: ${ }^{21}$ Solange sich der Staat innerhalb seines Erziehungsauftrags hält - und dies heißt: seine Maßnahmen auf neutrale Weise begründen kann -, ist es Sache der Vertreter der unterschiedlichen politischen, religiös-weltanschaulichen und ethischen Überzeugungen, sich mit den Wirkungen dieser Maßnahmen zu arrangieren. Das wird manchen Vertretern gut gelingen, auf der Grundlage anderer Überzeugungen kann es dagegen schwieriger sein; dies ändert aber nichts daran, dass niemand eine unfaire Benachteiligung geltend machen kann, solange nicht-diskriminierende Gründe für diese Maßnahmen vorgebracht werden können. Zugleich klärt dieser Ansatz das Verhältnis von staatlicher Erziehungsbefugnis und Elternrecht. Soweit sich die staatliche Schule innerhalb ihres Auftrags hält, ist das Elternrecht nicht berührt;

21 Zur Unterscheidung von Wirkungs- und Begründungsneutralität vgl. ausführlich HusteR (Anm. 3), S. 98 ff. und passim. 
es liegt - in der Terminologie der Grundrechtsdogmatik - kein Eingriff in das Elternrecht vor. Erst wenn die Schule die ihrem Auftrag immanenten und durch das Prinzip der Begründungsneutralität konkretisierten Grenzen überschreitet, entfaltet das Elternrecht seine Schutzwirkung. Diese Konstruktion hat den Vorteil, dass sie die Erziehungsbefugnisse in eindeutiger und sinnvoller Weise abschichtet. $^{22}$ Die Schule kann ihre neutral begründeten Erziehungsziele verfolgen, ohne auf die Zustimmung der Eltern angewiesen zu sein oder auch nur eine Abwägung mit deren kollidierenden Erziehungsvorstellungen vornehmen zu müssen. Dies vermeidet das von der herrschenden Abwägungstheorie weder offen ausgesprochene noch praktizierte, aber in ihrem Ansatz nur schwer vermeidbare Ergebnis, dass selbst elementare Erziehungsziele nicht oder nur eingeschränkt verfolgt werden dürfen, wenn sich genügend Eltern finden, die gegen diese Ziele Vorbehalte geltend machen. Dies heißt aber nicht, dass es sich dabei um ein striktes Separationsmodell handelt. Das Elternrecht wirkt vielmehr in doppelter Weise in die öffentliche Schule hinein. Zum einen ist es von Bedeutung, soweit die Schule über ihren neutral begründbaren Erziehungsauftrag hinausgehen will. Dies ist nicht prinzipiell ausgeschlossen, setzt aber die Zustimmung der betroffenen - und zwar aller - Eltern voraus. Das Elternrecht wirkt insoweit also kompetenzerweiternd. ${ }^{23}$ Zum anderen kann über das Elternrecht geltend gemacht werden, dass die Schule die ihr gesetzten Grenzen überschreitet; Art. 6 Abs. 2 GG subjektiviert insoweit das Neutralitätsprinzip. Wichtig ist dabei aber, dass damit eben objektive - d.h. von den subjektiven Vorstellungen der Eltern unabhängige - Grenzen des staatlichen Erziehungsauftrags geltend gemacht werden.

\section{Folgerungen für die Erziehung in der öffentlichen Schule}

Für die öffentliche Schule, in der der Staat unausweichlich mit den unterschiedlichen ethischen Überzeugungen der Bürger konfrontiert wird, ergeben sich aus diesem Ansatz mehrere Folgerungen.

Zunächst: Die schulische Erziehung ist zwar in ethischer Hinsicht, keineswegs aber in allen normativen Fragen auf eine neutrale Haltung verpflichtet. Soweit Grundsätze und Tugenden betroffen sind, die für das Zusammenleben in einem freiheitlichen Gemeinwesen als unentbehrlich oder zumindest för-

22 Ähnliche Konstruktion - allerdings auf der Grundlage eines erheblich engeren Erziehungsauftrags der Schule - bei Arnulf Schmitt-Kammler, Elternrecht und schulisches Erziehungsrecht nach dem Grundgesetz, 1983, S. 50 ff.

23 Dem entspricht im geltenden Verfassungsrecht etwa das Recht der Eltern, über die Teilnahme des Kindes am Religionsunterricht zu entscheiden (Art. 7 Abs. 2 GG). Vgl. näher dazu unten II. 7. b). 
derlich ausgewiesen werden können, steht es der Schule frei, diese Grundsätze und Tugenden affirmativ zu vertreten; nicht vorausgesetzt werden darf dagegen ein bestimmtes weltanschauliches oder ethisches Fundament. Neutralität kann daher - dies ist insbesondere gegenüber den karikierenden Behauptungen mancher Kritiker dieses Prinzips zu betonen - immer nur eine selektive Neutralität sein, deren näherer Umfang sich aus einer Anwendung des Gebots der Begründungsneutralität ergibt. Neutralität der Schule bedeutet demnach nicht umfassende ,Wertneutralität“ oder ,Wertfreiheit“, sondern selektive ethische Begründungsneutralität. Politische und sozialmoralische Erziehungsziele werden sich demnach eher rechtfertigen lassen als Erziehungsziele, die die Gestaltung des eigenen Lebens betreffen; dies gilt vor allem dann, wenn diese Ziele - wie etwa „Ehrfurcht vor Gott“ - auf einer partikularen religiös-weltanschaulichen Grundlage beruhen. Dass insbesondere ethische Erziehungsziele, die vorrangig die religiös-weltanschauliche Orientierung und das individuelle gute Leben betreffen, grundsätzlich in die familiäre Erziehung gehören, ist daher nicht Ursache, sondern Folge der staatlichen Neutralitätspflicht.

Eine ganz andere Frage ist es dagegen, ob die Schule gut beraten ist, besonderes Gewicht auf die Erziehung in normativen Fragen zu legen und wie diese Erziehung im einzelnen aussehen sollte. Vermutlich helfen Moralpredigten und dezidierte ,Werteerziehung“ wenig, intellektuelle Aufklärung und eine freiheitliche und gerechte Ausgestaltung des praktischen Zusammenlebens in der Schule dagegen sehr viel. Aber dies erfordert pädagogische und bildungspolitische Einschätzungen, für die weder das Verfassungsrecht noch sozialphilosophische Gerechtigkeitsprinzipien primär einschlägig sind.

Zudem: Die öffentliche Schule vertritt weder eine Weltanschauung, noch ist es ihre Absicht, die Schüler in eine kritische Distanz zu ihren religiös-weltanschaulichen Überzeugungen zu bringen oder sie gar von diesen Überzeugungen zu ,,befreien“. Trotzdem ist es natürlich möglich und sogar wahrscheinlich, dass sich auch die schulischen Maßnahmen, die durch neutrale Erwägungen gerechtfertigt werden können, in durchaus differenzierter Form auf die verschiedenen ethischen Überzeugungen und Lebensformen auswirken werden. So werden die Schüler schon allein dadurch, dass die Schule Fähigkeiten und Kenntnisse vermittelt und zu selbständigem Denken ermuntert, in die Lage versetzt, die Selbstverständlichkeit ihrer bisherigen Überzeugungen zu hinterfragen und sie mit alternativen Vorstellungen zu vergleichen. ${ }^{24}$ Lebensformen, die für ihre

24 Es ist sowohl von Befürwortern als auch von Kritikern eines Erziehungsauftrags der öffentlichen Schule behauptet worden, dass selbst ein politischer Liberalismus, der die Schule auf politische Erziehungsziele im engeren Sinne beschränkt, unweigerlich in einen ethischen Liberalismus umschlage, da sich politisch-liberale und ethisch-liberale Einstellungen und Haltungen in mancherlei Hinsicht entsprächen. Dies dürfte aber die Bedeutung 
Existenz auf ein homogenes ethisches Milieu oder einen weitgehenden Reflexionsverzicht angewiesen sind, wird dies möglicherweise nicht gut bekommen. Auch etwa die schulische Ermunterung, am öffentlichen und politischen Leben teilzunehmen - eine Grundvoraussetzung funktionierender demokratischer Ordnungen -, ,,benachteiligt" diejenigen Auffassungen, die diese Teilnahme aus religiös-weltanschaulichen Gründen ablehnen. Dies ist aber kein Einwand gegen das Neutralitätsgebot, sondern die Beschreibung eines unvermeidlichen Faktums: Keine politische Ordnung - und auch keine Schule - kann in dem Sinne neutral sein, dass sie sich auf alle Vorstellungen des Guten in gleicher Weise auswirkt, zu allen derartigen Vorstellungen in der gleichen Weise ,passt“. Deshalb geht es von vornherein lediglich um ein Gebot der Neutralität der Begründung staatlichen Handelns; eine Neutralität der Auswirkungen staatlichen Handelns ist dagegen weder möglich noch wünschenswert. ${ }^{25}$ Sie ist nicht möglich, weil eben jede Ordnung derartige Auswirkungen hat:

„Es gibt keine soziale Welt ohne Verluste, das heißt keine soziale Welt, die nicht einige Lebensformen ausschlösse, die auf ihre besondere Weise bestimmte grundlegende Werte verwirklichen. Jede Gesellschaft wird sich aufgrund ihrer Kultur und ihrer Institutionen mit einigen Lebensformen als unvereinbar erweisen. Aber diese gesellschaftlichen Unvermeidbarkeiten dürfen nicht als willkürliche Voreingenommenheit oder als Ungerechtigkeit mißverstanden werden. “26

Eine Wirkungsneutralität ist häufig auch gar nicht wünschenswert, weil der politische Liberalismus hofft, dass die liberalen Institutionen und Regeln die ethischen Überzeugungen und Lebensformen in einer Weise prägen und gegebenenfalls transformieren, dass sie mit den grundlegenden normativen Entscheidungen eines freiheitlichen Gemeinwesens vereinbar sind und diese sogar unterstützen. ${ }^{27}$ Der Einwand der Eltern, ihre Erziehungsabsichten würden behindert, wenn ihre Kinder in der Schule etwa mit anderen Lebensformen und Überzeugungen konfrontiert werden, wird daher grundsätzlich nicht gehört:

des Neutralitätsprinzips übersehen, aufgrund dessen die schulische Erziehung, die auf den Grundsätzen des politischen Liberalismus beruht, zu den ethischen Überzeugungen und Lebensformen als solchen gerade nicht Stellung nehmen darf. Zur weiteren Diskussion vgl. Eamonn Callan, Political Liberalism and Political Education, The Rev. of Politics 58 (1996), S. 5 ff.; Stephen G. Gilles, On Educating Children: A Parentalist Manifesto, The Univ. of Chicago LR 63 (1996), S. 937 ff.; Amy Gutmann, Civic Education and Social Diversity, Ethics 105 (1995), S. 557 ff.; StePhen Macedo, Transformative Constitutionalism and the Case of Religion. Defending the Moderate Hegenomy of Liberalism, Political Theory 26 (1998), S. 56 ff.; Nomi Maya Stolzenberg, „He Drew a Circle that Shut Me out": Assimilation, Indoctrination, and the Paradox of a Liberal Education, Harvard LR 106 (1993), S. $581 \mathrm{ff}$.

25 So jetzt auch Jestaedt (Anm. 13), $\ 156, \mathrm{Rn} .71 \mathrm{ff}$.

26 John Rawls, Die Idee des politischen Liberalismus, 1992, S. 383.

27 Vgl. dazu Macedo (Anm. 24), S. 56 ff. 
Unter der Voraussetzung, dass die pädagogische und bildungspolitische Prämisse nachvollziehbar ist, es gehöre zu den Aufgaben der Schule, die heranwachsenden Bürger über die ethische Pluralität in unserem Gemeinwesen zu informieren, ist es Sache der Eltern, die von diesem Unterricht ausgehenden Wirkungen mit ihren Erziehungsvorstellungen zu arrangieren.

Ferner: Dies hat Konsequenzen für die Forderung nach einer - was immer das auch sein mag - multikulturellen Ausgestaltung der öffentlichen Schule. ${ }^{28}$ Um alle Bürger in die öffentliche Schule zu integrieren und sie nicht in eine Abwehrhaltung - oder in Privatschulen - zu drängen, mag es gesellschafts- und bildungspolitisch klug sein, kulturellen Besonderheiten auch in der Schule großzügig entgegenzukommen und nicht alle Verpflichtungen - z.B. zur Teilnahme am Sexualkundeunterricht oder zur Teilnahme muslimischer Mädchen am Sport- und Schwimmunterricht - ausnahmslos durchzusetzen. Ein Anspruch auf eine derartige Befreiung besteht aber nur dann, wenn der Schüler sonst in einen unzumutbaren Gewissenskonflikt geriete; der Umstand allein, dass ein bestimmter Unterricht mit den Erziehungsvorstellungen und der Lebensform der Eltern kollidiert - und seien diese auch religiös begründet -, ist dagegen unbeachtlich. Keine gesellschaftliche Gruppe hat einen Anspruch darauf, dass sich die öffentliche Schule gerade an ihren kulturellen und ethischen Bedürfnissen orientiert; jeder Bürger hat nur einen Anspruch darauf, dass die schulischen Maßnahmen nicht auf einer partikularen weltanschaulichen oder ethischen Grundlage beruhen, also prinzipiell nicht neutral gerechtfertigt werden können. Die Beurteilung, ob eine Rechtfertigung in diesem Sinne auf guten politischen Gründen beruht, wird gelegentlich von kulturellen und ethischen Überzeugungen imprägniert sein, und insoweit wird sich die jeweilige Mehrheitskultur mit ihrem Verständnis durchsetzen. Eine kulturelle Minderheit mag dies als strukturelle Diskriminierung und Differenzblindheit eines nur scheinbar allgemeinen und neutralen Rechts kritisieren. Das Verfassungsrecht kann aber aus prinzipiellen Gründen nur tatbestandlich umgrenzte Freiheitsrechte gewähren und rechtliche Diskriminierungen verhindern; es kann - zumal in einer pluralistischen oder gar multikulturellen Gesellschaft - nicht umfassend dafür sorgen, dass die Rechtsordnung und die staatlichen Institutionen allen Lebensformen und Überzeugungen in gleicher Weise entsprechen. Dies müssen - soweit dies überhaupt möglich ist - vielmehr der politische Prozess und die öffentliche Diskussion leisten, indem sich dort die bisher vernachlässigten und übersehenen

28 Grundsätzlich zur Diskussion des Multikulturalismus vgl. die Beiträge in Amy GutmanN (Hrsg.), Multikulturalismus und die Politik der Anerkennung, 1993 (dort insbesondere Jürgen Habermas, Anerkennungskämpfe im demokratischen Rechtsstaat, S. 147 ff.); Will Kymlicka, Multikulturalismus und Demokratie. Über Minderheiten in Staaten und Nationen, 1999. 
Interessen und Bedürfnisse artikulieren. Die Verfassung und die Grundrechte haben insoweit natürlich wiederum eine enorme Bedeutung, als sie die Offenheit dieser Prozesse sicherstellen. Aber das Verfassungsrecht kann die politischen und gesellschaftlichen Anerkennungskämpfe, die sich in diesen Foren abspielen, nur ermöglichen, nicht jedoch advokatorisch vorwegnehmen. Schon deshalb kann es keinen Anspruch auf eine multikulturelle Schule geben.

Schließlich: Das Prinzip der Begründungsneutralität erlaubt eine sinnvollere Zuordnung von elterlicher und schulischer Erziehung, als sie die herrschende Abwägungs- und Gleichordnungstheorie vornimmt. Letztere beruht auf der Prämisse, ein erzieherisches ,Zusammenwirken“ von Eltern und Schule sei geboten, weil nur auf diese Weise dem Umstand Rechnung getragen werden könne, dass sich die Erziehungsbemühungen auf die „Bildung der einen Persönlichkeit des Kindes“ auswirken. Dieser Position liegt mehr oder weniger deutlich die Vorstellung einer einheitlichen Persönlichkeit zugrunde, deren Entwicklung durch unterschiedliche oder gar einander widersprechende Erziehungsbemühungen gestört wird. Durch die Verpflichtung der Schule, mit den Eltern zu kooperieren und ihren „Gesamterziehungsplan“ zu achten, sollen derartige Erziehungskonflikte vermieden oder zumindest weitestgehend abgeschwächt werden. Dementsprechend ist auch gegen Versuche, elterliche und schulische Erziehungsbefugnisse einander überschneidungsfrei zuzuordnen, geltend gemacht worden, eine derartige „Trennung“ sei dem Umstand nicht angemessen, dass „,elterliche wie schulische Erziehungsmaßnahmen ihren Effekt beim nämlichen Kind haben".

Es ist dann allerdings schwer verständlich, warum das Grundgesetz der öffentlichen Schule überhaupt eine Befugnis zur Erziehung zugewiesen und diese nicht vielmehr vollständig den Eltern überlassen hat. Die verfassungsrechtliche Entscheidung für eine erzieherische Gewaltenteilung zwischen Eltern und Schule setzt voraus, dass sich die öffentliche Schule nicht an den elterlichen Erziehungsvorstellungen orientieren muss, sondern einen eigenständigen Beitrag zur Persönlichkeitsbildung leisten darf, den die Eltern nicht oder zumindest nicht in gleicher Weise erbringen können. Wird dies aber zugestanden, so muss man davon ausgehen, dass die Verfassung unterschiedliche erzieherische Einflüsse auf das Kind in Kauf nimmt; auch ist es dann nicht mehr ausgeschlossen, dass sich elterlicher und schulischer Erziehungsauftrag strikt voneinander abgrenzen lassen. Die im Grundgesetz verankerte erzieherische Gewaltenteilung legt sogar im Gegenteil den Gedanken nahe, dass die Vorstellung, anzustreben und verfassungsrechtlich zu gewährleisten seien eine möglichst einheitliche Persönlichkeit des Educanden und folglich auch die weitgehende Übereinstimmung der Bemühungen verschiedener Erziehungsträger, grundsätzlich verfehlt ist: Das Grundgesetz nimmt die Unterschiedlichkeit der erzieherischen Einflüsse nicht 
nur in Kauf, sondern beabsichtigt sie sogar. Die Gegenüberstellung von elterlicher und schulischer Erziehung in Art. 6 Abs. 2 und Art. 7 Abs. 1 GG fordert nicht zu einer möglichst konfliktfreien Zuordnung in dem Sinne auf, dass im besten Falle die Erziehungsbemühungen und -absichten von Elternhaus und Schule identisch sind; vielmehr liegt der Sinn dieser Aufteilung gerade darin, dass die Persönlichkeit des Heranwachsenden sich ergänzenden, aber doch auch betont unterschiedlichen Erziehungsbemühungen ausgesetzt wird.

Den sachlichen Hintergrund dieser Auffassung bildet die Einsicht, dass nicht eine möglichst einheitliche, sondern allein eine vielgestaltige Erziehung den pädagogischen Erfordernissen in einem differenzierten und pluralistischen Gemeinwesen gerecht wird. Das Leben in diesem Gemeinwesen verlangt vor allem die Fähigkeit zur Rollendifferenzierung; in einer offenen Gesellschaft, die sich nicht durch ein hohes Maß an Homogenität, sondern durch einen ethischen Pluralismus auszeichnet, ist es zudem von zentraler Bedeutung, dass man mit den eigenen ethischen Überzeugungen und zugleich auf der Grundlage der Gleichberechtigung inmitten einer Vielzahl von Orientierungsmustern und Lebensformen zurechtzukommen lernt. Diese Anforderungen legen aber weniger das Erziehungsideal einer möglichst einheitlichen Persönlichkeit nahe, sondern verweisen auf die Vorstellung eines differenzierten Selbst, das in der Lage ist, diese Unterscheidungsleistungen zu erbringen. Dass eine allein von den Eltern geleistete oder ausschließlich an ihren Vorstellungen orientierte Erziehung diesem Ziel gerecht wird, ist nicht gewährleistet, in mancherlei Hinsicht sogar eher unwahrscheinlich: Denn die elterliche Erziehung wird sich häufig darauf konzentrieren oder sogar darauf beschränken, den Heranwachsenden in die eigene Lebensform einzuführen; es kann nicht ohne weiteres davon ausgegangen werden, dass dies eine ausreichende Vorbereitung für die Wahrnehmung anderer Rollen und für die Begegnung mit abweichenden Auffassungen darstellt. Das Gemeinwesen hat aber ein nachvollziehbares Interesse daran, dass die zukünftigen Bürger bereit und in der Lage sind, auch die Funktionen, die über den privaten und familiären Lebensbereich hinausgehen, in sozialverträglicher Weise auszufüllen und sich in einer pluralistischen Gesellschaft zurechtzufinden. Aus dieser Perspektive der schulischen Integrationsaufgabe ist es kein Nachteil, sondern gerade erwünscht, dass der elterliche Einfluss auf die Kinder durch die Erziehung in der öffentlichen Schule ergänzt und gegebenenfalls auch zurückgedrängt und korrigiert wird. Die Kinder sind weder Eigentum ihrer Eltern noch Eigentum des Staates; sie sind weder nur Familienmitglieder noch ausschließlich ,öffentliche“ Personen. Eben darin liegt der Sinn der erzieherischen Gewaltenteilung:

„[...] neither parents nor a centralized state have a right to exclusive authority over the education of children. Because children are members of both families and states, the educational authority of parents and of polities has to be partial to be justified. [...] Parents 
acting individually and citizens acting collectively both have valuable and largely complementary roles to play in the moral education of children: the former in teaching children what it means to be committed to particular people and one way of life among many; the latter in teaching responsibilties and rights within a larger and more diverse community. “29

Es kann daher von vornherein nicht darum gehen, mögliche Spannungen zwischen elterlicher und schulischer Erziehung aufzulösen, indem die Schule ihre Erziehungsziele den elterlichen Vorstellungen so weit wie möglich anpasst. Aufgabe muss vielmehr eine Erziehung sein, die dazu befähigt, diese Spannungen und unterschiedlichen Erwartungshaltungen auszuhalten und sinnvoll mit ihnen umzugehen. Das Prinzip der Begründungsneutralität reflektiert die erzieherische Gewaltenteilung und ihre soziale Funktion. Es gewährt den Eltern im Bereich der familiären Erziehung einen weitgehenden Gestaltungsfreiraum und beschränkt den Staat auf eine Missbrauchskontrolle. Der Verzicht, den Eltern im Interesse der Rechts- und Sozialgemeinschaft inhaltliche Erziehungsvorgaben aufzuerlegen, wird dadurch kompensiert, dass die öffentliche Schule nicht auf eine umfassende Wertneutralität, sondern auf eine selektive ethische Begründungsneutralität verpflichtet wird, die ihr eine integrationsorientierte Erziehung und damit die Vermittlung der normativen Grundlagen erlaubt, die das politische und gesellschaftliche Zusammenleben bestimmen. Die Eltern können daher eine erzieherische Beeinflussung durch die Schule abwehren, die sich unmittelbar gegen die ethischen Überzeugungen und die Lebensform richtet, die sie ihren Kindern vermitteln wollen; eine schulische Erziehung zu politischer und sozialer Kompetenz müssen sie dagegen auch dann hinnehmen, wenn diese die Wirkung besitzt, ihre eigenen Erziehungsbemühungen zu erschweren oder gar zu vereiteln.

\section{Schulische „Werteerziehung“ im Lichte des Neutralitätsgebots}

Das im Blick auf die staatliche Neutralitätsverpflichtung besonders heikle Ansinnen, in der öffentlichen Schule eine eigenständige und entschiedene ,Werteerziehung“ zu installieren, hat insbesondere die jüngeren Diskussionen um den Religionsunterricht, den Ethikunterricht - oder welche Bezeichnung dieses Fach in den einzelnen Bundesländern auch tragen mag - und das Verhältnis dieser Fächer geprägt. Anhand dieser Problematik soll abschließend angedeutet werden, zu welchen Folgerungen das hier entwickelte Konzept der verfassungs-

29 Diese ist eine der Grundthesen des vorzüglichen Buches von Amy Gutmann, Democratic Education, 2. Aufl. 1999, S. 30 und 54. 
rechtlichen Zulässigkeit und der verfassungsrechtlichen Grenzen der schulischen Erziehung führt.

\section{a) Ethikunterricht in der öffentlichen Schule}

Nach dem Prinzip der selektiven Begründungsneutralität ist ein Ethikunterricht, der auf die eine oder andere Weise eine „Werteerziehung“ beabsichtigt, grundsätzlich zulässig. Verfassungsrechtliche Vorgaben ergeben sich allerdings zunächst für die Frage, welche ,Werte“ den Schülern nahegelegt werden dürfen: Es muss sich um Orientierungen handeln, die nicht auf einer partikularen ethischen Grundlage beruhen, sondern als allgemein nachvollziehbar dargestellt werden müssen. Eher theoretische als praktische Probleme werfen insoweit die deutlich christlich imprägnierten Formulierungen in einigen Landesverfassungen und Landesschulgesetzen auf. Das Bundesverfassungsgericht hat derartige religiös-weltanschauliche Färbungen in seiner Rechtsprechung zur ,christlichen Gemeinschaftsschule“ grundsätzlich akzeptiert, gleichzeitig aber in einer verfassungskonformen Auslegung auf den Status von unverbindlichen Elementen der kulturellen Tradition reduziert. ${ }^{30}$ Dass damit nur ein dilatorischer Formelkompromiss erreicht war, wurde 20 Jahre später in der Schulkreuz-Entscheidung offenbar, die die staatliche Neutralitätspflicht dann - jedenfalls im Ergebnis ${ }^{31}$ unmissverständlich zur Geltung gebracht hat. ${ }^{32}$

Weniger aussagekräftig ist das Verfassungsrecht dagegen zu der Frage, wie ein Ethikunterricht methodisch auszurichten ist. Diese Frage stellt sich insbesondere, wenn nach der jeweiligen Unterrichtskonzeption nicht lediglich über moralische Normen und Haltungen informiert, ein Reflexionsprozess angeregt und ein Zugang zu moralischen Fragen eröffnet werden, sondern auf der Grundlage eines moralpädagogischen Modells im engeren Sinne auch ein Beitrag zur moralischen Erziehung geleistet werden soll, indem versucht wird, den Schülern bestimmte Normen und Haltungen zu vermitteln. Gegen dieses Modell, das in den derzeitigen Ausgestaltungen des Ethikunterrichts durchaus vorherrschend sein mag, sind aus philosophischer und pädagogischer Sicht beachtliche Bedenken vorgetragen worden. ${ }^{33}$ Das Dilemma der Wertevermittlung, die hier angestrebt werde, liege nicht nur darin, dass der Ethikunterricht auf den moralischen Aspekt verengt werde; vor allem sei ein Schulfach mit der Vermittlung sittlicher Kompetenz prinzipiell überfordert: Der Ethikunterricht könne nämlich im We-

30 Vgl. BVerfGE 41, S. 29 ff.; 41, S. 65 ff.; 41, S. 88 ff.

$31 \mathrm{Zu}$ den Begründungsschwächen der Entscheidung vgl. Huster (Anm. 3), S. $127 \mathrm{ff}$.

32 BVerfGE 93, S. 1 ff.

33 Vgl. dazu nur OtFried HöfFe, Ethikunterricht in pluralistischer Gesellschaft, in: ders. (Hrsg.), Ethik und Politik, 1979, S. 453; Georg Lohmann, Probleme der „Werteerziehung“ im Ethikunterricht, Deut. Zeitschr. für Philosophie 46 (1998), S. 291 ff. 
sentlichen nur in theoretisch orientierten Lernprozessen moralische Einsichten erzeugen; es sei aber keineswegs gewährleistet, dass sich diese Einsichten auch in moralischen Haltungen und Handlungen niederschlagen. Ethos sei eben nicht lehrbar; im schlimmsten Falle begünstige man durch die intellektualistische Verkürzung der sittlichen Kompetenz die Heuchelei. Die erwünschte Kompetenz bilde sich allein durch die Teilnahme an einer sittlichen Praxis; zu dieser könne auch die Gestaltung des Ethikunterrichts beitragen, ohne dass ihn dies aber gegenüber den anderen Schulfächern auszeichne - und, wie man hinzufügen muss, ohne dass sich die Verwirklichung dieser Idealvorstellung rechtlich garantieren ließe. Aus der Beobachtung dieser Kluft zwischen theoretischer sittlicher Einsicht und praktischer sittlicher Kompetenz ziehen die Kritiker des moralpädagogischen Modells die Konsequenz, das Lernziel des Ethikunterrichts müsse sich auf die Vermittlung der Fähigkeit beschränken, moralische Probleme zu erkennen und zu artikulieren, um auf diese Weise das reflexive Urteilsvermögen im Sinne einer „Hilfe zur Selbsthilfe“ und der Förderung von „Orientierungsautonomie" auszubilden.

Für dieses „Nachdenklichkeits-Modell“ mögen in der Tat gute pädagogische Gründe sprechen. Die Radikalität der zugrunde liegenden Kritik mag man allerdings für übertrieben halten; die Alltagserfahrung legt eher die Einschätzung nahe, dass Einsicht immerhin der erste Schritt zur Besserung sein kann. Zudem schließen sich Nachdenklichkeit und die Vermittlung von moralischen Normen und Haltungen wohl nicht schlechthin aus. Wie dem auch sei: Eine verfassungsrechtliche Verpflichtung für den Gesetzgeber, den Ethikunterricht im Sinne dieses „Nachdenklichkeits-Modells“ auszugestalten und insoweit auf die moralerzieherische Funktion im engeren Sinne vollständig oder weithin zu verzichten, besteht nicht. Denn das Grundgesetz enthält kein verbindliches pädagogisches Programm: Ob der Ethikunterricht eine „,formale BewertungsErziehung“ oder eine „,materiale Werteinstellungs-Erziehung“ anstrebt, ${ }^{34}$ liegt in der Gestaltungsfreiheit des Gesetzgebers, die notwendigerweise mit dem eigenständigen Erziehungsauftrag der Schule verbunden ist. Aufgrund des Persönlichkeitsrechts der Schüler existiert zwar ein verfassungsrechtliches Verbot der Indoktrination; und ein moralpädagogischer Ethikunterricht mit zudem starker „lebenskundlicher“ Prägung mag stärker der Gefahr ausgesetzt sein, mit diesem Verbot in Konflikt zu geraten, als ein an dem „Nachdenklichkeits-Modell“ ausgerichtetes Fach mit ganz überwiegend kognitiven Anteilen. Aber es wäre unsinnig, jeden Versuch der Vermittlung von Normen und Haltungen in der Schule als Indoktrination zu diffamieren, und so lässt die Verfassung dem

34 So die Gegenüberstellung bei Wolfgang Brezinka, Werterziehung? Problematik und Möglichkeiten, in: ders., Glaube, Moral und Erziehung, 1992, S. 142, 159 ff. 
Gesetzgeber und der Schule einen weitreichenden Gestaltungsspielraum. Die Diskussion um die Gestaltung des Ethikunterrichts bestätigt damit, dass die verfassungsrechtliche Betrachtung sich nicht primär auf die Methoden, sondern auf die Inhalte konzentrieren sollte, die in der staatlichen Schule vermittelt werden. Mit dem erwähnten Indoktrinations-Vorbehalt wird man daher sagen können, dass auch gegen einen dezidiert moralpädagogisch orientierten Ethikunterricht in der staatlichen Schule keine verfassungsrechtlichen Bedenken bestehen, soweit er inhaltlich dem Neutralitätsprinzip genügt.

\section{b) Religionsunterricht in der öffentlichen Schule}

Das Neutralitätsprinzip verbietet der öffentlichen Schule, die eine „Werteerziehung" beabsichtigt, dieser Erziehung eine verbindliche religiös-weltanschauliche Grundlage zugrunde zu legen. Ob eine „Werteerziehung“ ohne ein derartiges Fundament überhaupt sinnvoll möglich ist, ist häufig bezweifelt worden. ${ }^{35} \mathrm{Zwar}$ sollte man angesichts der Notwendigkeit, sich in einer pluralistischen Gesellschaft auch dann über verbindliche Normen des Zusammenlebens zu einigen, wenn man in ethischen Fragen nicht übereinstimmt, mit dieser Skepsis zurückhaltend sein; aber man kann der Ansicht sein, dass eine „Werteerziehung“ ohne weltanschauliches Fundament letztlich einer Frau ohne Unterleib ähnelt. Die verfassungsrechtlich gebotene Zurückhaltung der öffentlichen Schule in religiösweltanschaulichen Angelegenheiten hat die Suche nach Formen der schulischen Erziehung bestärkt, die auf eine „dichtere“ ethische Grundlage zurückgreifen können. Dies betrifft - neben den Privatschulen - den konfessionellen Religionsunterricht.

Liberale haben gegenüber dem Religionsunterricht als ordentlichem Lehrfach und der damit verbundenen Kooperation von Staat und Religionsgemeinschaften häufig Vorbehalte geäußert und diese auch mit dem Neutralitätsgebot begründet. Juristisch drückte sich dies darin aus, dass die Garantie dieses Unterrichts in Art. 7 Abs. 3 GG als prinzipienwidriges oder sogar verfassungswidriges Verfassungsrecht eingeordnet wurde. ${ }^{36}$ Wenn man das Neutralitätsgebot im Sinne des Prinzips der Begründungsneutralität versteht, ist dies jedoch nicht unmittelbar einsichtig. Mit dem konfessionell ausgerichteten Religionsunterricht ist ein Erziehungselement in die öffentliche Schule integriert, das der Schule selbst aufgrund ihrer Neutralitätsverpflichtung nicht zur Verfügung steht. Dagegen ist aus Sicht des Neutralitätsprinzips wenig einzuwenden, wenn die folgenden Be-

35 Vgl. etwa die Beiträge in Christoph Gestrich (Hrsg.), Ethik ohne Religion?, Beiheft zur Berliner Theologischen Zeitung, 1996.

36 Vgl. etwa Ludwig ReNCK, Rechtsfragen des Religionsunterrichts im bekenntnisneutralen Staat, DÖV 1994, S. 27 ff. 
dingungen erfüllt sind, die in Art. 7 Abs. 2 und 3 GG auch tatsächlich gewährleistet werden: Die Teilnahme an diesem Unterricht muss freiwillig sein; der Staat darf nicht selbst die zu vermittelnden Glaubenssätze bestimmen, sondern muss dies den Religionsgemeinschaften überlassen; und der Zugang zum Religionsunterricht muss grundsätzlich allen Religionsgemeinschaften offen stehen. Wenn dies der Fall ist, ist nicht ersichtlich, inwieweit der Religionsunterricht eine Kompromittierung des Neutralitätsgedankens darstellt. Allerdings setzt dies voraus, dass man das Neutralitätsgebot nicht mit einer strikten Trennung von Staat und Religionsgemeinschaften identifiziert. Dafür besteht aber auch keine Notwendigkeit: $\mathrm{Ob}$ und inwieweit in staatlichen Institutionen derartige religiösweltanschaulich geprägte Sachverhalte einen Platz haben, ist regelmäßig eine Frage der politischen Tradition und der gesellschafts- und bildungspolitischen Klugheit, berührt aber nicht - solange die genannten Bedingungen erfüllt sind die grundlegenden Gerechtigkeitsprinzipien des Gemeinwesens.

c) Das Verhältnis von Religions- und Ethikunterricht

Verfassungsrechtliche Fragen werfen daher auch weniger Ethik- und Religionsunterricht als solche auf; problematisch ist eher das Verhältnis dieser Fächer zueinander.

Dies betrifft zunächst die in einigen Bundesländern bestehende Regelung, nach der die nicht am Religionsunterricht teilnehmenden Schüler zum Besuch eines Ethikunterrichts verpflichtet sind, um das Defizit an „,Werteerziehung“ auszugleichen. Diese Verpflichtung ist mit dem Argument angegriffen worden, sie beeinträchtige die Freiwilligkeit der Teilnahme am Religionsunterricht. ${ }^{37}$ $\mathrm{Ob}$ dies zutreffend ist, ob also der Ethikunterricht als ein unzulässiger ,Strafunterricht für Religionsunterrichtsverweigerer" angesehen werden muss, hängt gemäß dem Grundsatz der Begründungsneutralität davon ab, ob eine neutrale Rechtfertigung für dieses Ersatzfach gegeben werden kann. Diese Rechtfertigung kann sich allein aus dem Bildungs- und Erziehungsauftrag der öffentlichen Schule ergeben: Wenn der Religionsunterricht der religiös-weltanschaulichen Bildung und Erziehung dient, so ist die Überlegung, dass die Schüler, die - aus welchen Gründen auch immer - nicht am Religionsunterricht teilnehmen, auf andere Weise in diesem Bereich schulisch betreut werden sollten, aus Sicht des Neutralitätsprinzips grundsätzlich nicht zu beanstanden. Dass damit der eine oder andere Schüler tatsächlich zur Teilnahme am Religionsunterricht bewegt wird, ist lediglich die unbeachtliche Auswirkung einer an sich neutralen Maß-

37 Vgl. etwa Gerhard Czermak, Das Pflicht-Ersatzfach Ethikunterricht als Problem der Religionsfreiheit, des Elternrechts und der Gleichheitsrechte, NVwZ 1996, S. 450 ff.; Ludwig RenCK, Verfassungsprobleme des Ethikunterrichts, BayVBl 1992, S. 519 ff. 
nahme und deshalb - wie es jetzt das Bundesverwaltungsgericht formuliert hat - eine zulässige „,mittelbare Einflussnahme“.38

Diese Rechtfertigung steht aber unter der Voraussetzung, dass das jeweilige Ersatzfach das gleiche Gewicht wie der Religionsunterricht besitzt, damit die Entscheidungsfreiheit der Betroffenen nicht durch eine „,curriculare Minderausstattung" beeinträchtigt wird. Dies mag in der Praxis nicht immer der Fall sein, kann aber durch das jeweilige Landesrecht ohne grundsätzliche Schwierigkeiten sichergestellt werden. Darüber hinaus ist es erforderlich, dass das Ersatzfach dem Religionsunterricht inhaltlich weithin entspricht; ein Fach mit ,,thematisch völlig anderen Erziehungs- und Bildungszielen" könnte nicht mehr über den Erziehungs- und Bildungsauftrag der Schule gerechtfertigt werden und wäre daher als unzulässige Beeinträchtigung der Freiwilligkeit der Teilnahmeentscheidung zu qualifizieren. Die Zulässigkeit der Einführung eines Ersatzfachs dürfte sich insoweit an der Frage entscheiden, welche Unterschiede zwischen diesem Fach und dem Religionsunterricht toleriert werden können: Denn eine inhaltliche Identität zwischen diesen Fächern wird es schon deshalb nicht geben, weil es den Religionsunterricht gerade auszeichnet, nicht nur zu sagen, ,was geglaubt wird, sondern auch, was geglaubt werden soll"“; dies ist dem neutralen Ethikunterricht aber von vornherein verwehrt. Trotzdem werden sich aus dieser unvermeidlichen Differenz der beiden Fächer keine durchgreifenden Bedenken herleiten lassen. Die Integration des Religionsunterrichts in die öffentliche Schule beruht auf der nachvollziehbaren Begründung, dass eine religiös-weltanschauliche Erziehung so weit wie möglich aus der Binnenperspektive erfolgen sollte. Wenn man dies aber akzeptiert, liegt es in der Natur der Sache, dass ein von der staatlichen Schule inhaltlich verantwortetes Ersatzfach „Ethik“ sich von diesem Unterricht insoweit unterscheidet.

In umgekehrter Blickrichtung stellt sich die Frage, ob und inwieweit der Religionsunterricht durch einen obligatorischen Ethikunterricht ersetzt oder ergänzt werden darf. Die Bundesländer sind durch Art. 7 Abs. 3 S. 1 GG zur Einrichtung des Religionsunterrichts als ordentliches Lehrfach grundsätzlich verpflichtet; ob sich auf die Ausnahmeklausel des Art. 141 GG, die jedenfalls für Bremen und Berlin gilt, auch die neuen Bundesländer berufen können, ist umstritten; das Bundesverfassungsgericht hat mit seinem Vergleichsvorschlag zum LER-Konzept eine Klärung dieser Frage umgangen. ${ }^{39}$

Wenn Art. 7 Abs. 3 S. 1 GG in einem Bundesland nicht anwendbar ist wie in Bremen und Berlin, gegebenenfalls auch in den neuen Bundesländern -, insoweit also keine Verpflichtung zur Einrichtung eines Religionsunterrichts

38 BVerwG DÖV 1998, S. 1058, 1061.

39 Vgl. BVerfGE 104, S. 305 ff. 
besteht, ist es zunächst umstritten, ob dieses Bundesland trotzdem berechtigt ist, den Religionsunterricht als ordentliches Lehrfach einzuführen. Dies betrifft etwa die Diskussion in Berlin: Hier wird an den Schulen bisher nur ein Religionsunterricht angeboten, der von den Religionsgemeinschaften in eigener Regie und ohne staatliche Beteiligung durchgeführt wird. Da sich nun auch islamische Vereinigungen auf diese Möglichkeit berufen, die das Berliner Schulgesetz gewährt, gab und gibt es politische Bestrebungen, sich der Rechtslage in den anderen Bundesländern anzunähern und den Religionsunterricht in staatlicher Verantwortung durchzuführen, um das Aufsichtsrecht gegenüber einer problematischen - ,fundamentalistischen“ und verfassungsfeindlichen - Ausgestaltung dieses Unterrichts in Stellung bringen zu können. Folgt man der Ansicht, dass auch ein Religionsunterricht als ordentliches Lehrfach trotz der notwendigen Kooperation von Staat und Religionsgemeinschaften nicht gegen das Neutralitätsgebot verstößt, ist gegen diese Pläne grundsätzlich nichts einzuwenden. Dass Art. 7 Abs. 3 S. 1 GG in Berlin nicht anwendbar ist, führt also nur dazu, dass der ordentliche Religionsunterricht nicht eingeführt werden muss; es führt nicht dazu, dass er nicht eingeführt werden darf. Das Land Berlin hat damit alle Freiheiten: Es kann einen ordentlichen Religionsunterricht einführen, es bei der bisherigen Regelung eines allein von den Religionsgemeinschaften verantworteten Unterrichts belassen oder auch gar keinen Religionsunterricht in der Schule vorsehen.

Die letztgenannte Möglichkeit, auf einen Religionsunterricht unter Beteiligung der Religionsgemeinschaften vollständig $\mathrm{zu}$ verzichten, ist allerdings in ihrer verfassungsrechtlichen Zulässigkeit nicht ganz unumstritten. So ist in der Auseinandersetzung um das Brandenburger Modell, den Religionsunterricht durch das Fach LER (Lebensgestaltung - Ethik - Religionskunde) zu ersetzen, gelegentlich angedeutet worden, auch unabhängig von Art. 7 Abs. 3 GG sei der Staat verpflichtet, eine religiös-weltanschauliche Unterweisung in der Schule $\mathrm{zu}$ ermöglichen, die aus der Binnenperspektive der Religionsgemeinschaften erfolgt. ${ }^{40}$ Soweit in diesem Zusammenhang auf die Religionsfreiheit der Eltern, Schüler und Religionsgemeinschaften verwiesen wird, ist das kaum plausibel, denn aus diesem Grundrecht ergibt sich schwerlich ein Anspruch, dass der Religionsunterricht gerade als ordentliches Lehrfach in die öffentliche Schule integriert werden muss. Der heikle Punkt ist allerdings auch etwas anders gelagert: Eltern, Schüler und Religionsgemeinschaften wehren sich gegen die Verpflichtung zum Besuch eines LER-Unterrichts, in dessen religionskundlichen Anteilen ihre religiös-weltanschauliche Überzeugung nicht aus der Binnenper-

40 So etwa Martin Heckel, Religionskunde im Lichte der Religionsfreiheit, ZevKR 44 (1999), S. $147 \mathrm{ff}$. 
spektive, sondern aufgrund der Neutralitätsverpflichtung dieses Unterrichts nur „,von außen“ dargestellt werden kann. Dass das Land Brandenburg die Teilnahmeverpflichtung für diejenigen Schüler, die eine außerschulische religiöse Unterweisung besuchen, zunächst suspendiert hat, ändert nichts an der Grundfrage: Folgt aus der Einrichtung eines Schulfachs, das der „Werteerziehung“ dienen soll - ob es nun Ethikunterricht oder LER genannt wird -, die staatliche Verpflichtung, den religiös-weltanschaulich gebundenen Schülern ein gleichwertiges Alternativangebot zur Verfügung zu stellen, das eine „Werteerziehung“ aus der Binnenperspektive ihrer jeweiligen Überzeugung ermöglicht? Dieses Problem tritt übrigens nicht nur in den Bundesländern auf, in denen kein Religionsunterricht existiert; ebenso kann man fragen, ob die anderen Bundesländer berechtigt wären, den Ethikunterricht nicht nur - wie bisher - als Ersatzfach für „Religionsunterrichtsverweigerer“, sondern als obligatorisches Fach für alle Schüler vorzusehen.

Aus der Sicht eines Abschichtungsmodells, das sich am Grundsatz der Begründungsneutralität orientiert, wird man zu dem Ergebnis gelangen müssen, dass die öffentliche Schule den Schülern zumuten darf, einen ,wertorientierten" Unterricht zu besuchen, der nicht auf ihren jeweiligen Überzeugungen beruht. Es ist ein legitimes bildungspolitisches Anliegen, die Schüler über die unterschiedlichen religiösen und weltanschaulichen Positionen zu informieren, die in unserem Gemeinwesen vertreten werden, um ihre Orientierungs- und Toleranzfähigkeit zu stärken. Da dieser Unterricht von allen Schülern besucht werden soll, ist es unausweichlich, dass er zu den dargestellten Überzeugungen eine sachliche und insoweit distanzierte Einstellung einnehmen muss. Es mag für den einen oder anderen Schüler schwer erträglich sein, dass auch seine Überzeugung auf diese Weise „von außen“, als eine von mehreren Möglichkeiten und ohne ihren konstitutiven Verbindlichkeitsanspruch dargestellt wird. Dies ist aber in einer freiheitlichen und pluralistischen Gesellschaft und ihrer Schule zu ertragen; solange dieser Unterricht nicht versucht, die Schüler von ihren Überzeugungen abzubringen oder diese zu „vergleichgültigen“ - obwohl natürlich nie ganz ausgeschlossen werden kann, dass ein Unterricht diese Wirkungen hat -, ist der Einwand mangelnder Neutralität nicht gerechtfertigt. ${ }^{41}$

Wiederum auf einer anderen Ebene kann man bezweifeln, ob ein derartiger Unterricht sinnvoll ist und nicht nur ein beliebiges Angebot der Religionen und Weltanschauungen präsentieren kann, das den Schüler völlig orientierungslos

41 Der jedenfalls in seiner Allgemeinheit unzutreffende Ausspruch eines brandenburgischen Abgeordneten „Was Werte sind, bestimmen wir“ hat zu dem Eindruck beigetragen, dass der LER-Unterricht diesen Anforderungen nicht genügt. Das brandenburgische Schulgesetz ist insoweit allerdings unmissverständlich und betont die religiös-weltanschauliche Neutralität; alles Weitere hängt von der näheren Ausgestaltung dieses Unterrichts ab. 
zurücklässt. Sollte eine ,Werteerziehung“ nur auf einer entschiedenen religiösweltanschaulichen Grundlage möglich sein, so spräche dies eher dafür, die Religionsgemeinschaften so weit wie möglich in die Schule zu integrieren und etwa einen Wahlpflichtbereich einzurichten, in dem die Schüler zwischen Religionsund Ethikunterricht wählen können. Für die Entscheidung zwischen diesen Alternativen wird viel davon abhängen, ob es gelingt, ein Fach zu konzipieren, das die Behandlung normativer Fragen in neutraler, jedoch nicht beliebiger Weise ermöglicht. Aber dies sind pädagogische und bildungspolitische Fragen, zu denen die Verfassung schweigt.

\section{Staatliche Gesundheitserziehung zwischen Paternalismus und Autonomieförderung}

\section{Auf dem Weg zur Gesundheitserziehungsdiktatur?}

Ein weiteres Politikfeld, in dem Vorbehalte gegen staatliche Erziehungsambitionen gelegentlich recht aggressiv formuliert werden, ist die Gesundheitspolitik. ${ }^{42}$ Inzwischen hat man auch in Deutschland ansatzweise erkannt, dass das System der medizinischen Versorgung weder für die Gesundheit der Bevölkerung - traditionell formuliert: die Volksgesundheit - noch für die soziale Verteilung von Gesundheit - die „Gesundheitsgerechtigkeit“ - von zentraler Bedeutung ist. Tatsächlich weisen zahlreiche Untersuchungen darauf hin, dass für den Gesundheitszustand einer Population maßgeblich andere, nämlich im weitesten Sinne soziale Faktoren verantwortlich sind, nämlich - neben dem Zugang zur Gesundheitsversorgung und der genetischen Konstitution - die Verhältnisse am Arbeitsplatz und im Wohnumfeld, das individuelle Gesundheitsverhalten sowie originär sozialstrukturelle Faktoren. Diese Faktoren dürften auch maßgeblich den sozialen Gesundheitsgradienten erklären. ${ }^{43}$ Es lässt sich nämlich nachweisen,

42 Vgl. zum Folgenden auch Stefan Huster, Eigenverantwortung im Gesundheitsrecht, in: Alena Buyx/Stefan Huster (Hrsg.), Ethische Aspekte von Public Health, Sonderheft von Ethik in der Medizin, Heft 3/2010, S. 289 ff.; DERs., Soziale Gesundheitsgerechtigkeit, 2011.

43 Vgl. dazu Johannes Siegrist/Michael Marmot (Hrsg.), Soziale Ungleichheit und Gesundheit: Erklärungsansätze und gesundheitspolitische Folgerungen, 2008; SACHVERStÄndigenRat zur Begutachtung Der Entwicklung im Gesundheitswesen, Gutachten 2005: Koordination und Qualität im Gesundheitswesen, 2006, Tz. 111 ff.; Ulrich Bauer u.A. (Hrsg.), Health Inequalities. Determinanten und Mechanismen gesundheitlicher Ungleichheit, 2008; Matthias Richter/Klaus Hurrelmann (Hrsg.), Gesundheitliche Ungleichheit. Grundlagen, Probleme, Perspektiven, 2. Aufl. 2009; Andreas Mielck, Soziale Ungleichheit und Gesundheit, 2005. 
dass diese gesundheitsrelevanten Faktoren zu einem erheblichen Teil schichtenspezifisch ausgeprägt sind: Angehörige der unteren sozialen Schichten sind - wie die einschlägigen Studien zeigen - häufiger einem gesundheitlich belastenden Arbeitsumfeld ausgesetzt und leiden auch in ihrem Wohnumfeld häufiger unter Lärm und Luftverschmutzung; sie ernähren sich ungesünder, neigen stärker zu Nikotinkonsum und vernachlässigen die körperliche Bewegung; schließlich leiden sie sowohl in ihrem Privat- und Arbeitsleben als auch in der (politischen) Öffentlichkeit an geringen Kontroll- und Selbstbestimmungsmöglichkeiten. Jedenfalls können die sozialen Gesundheitsungleichheiten zumindest in Deutschland gewiss nicht ausschließlich auf den Unterschied von gesetzlicher und privater Krankenversicherung oder auf soziale Zugangsbarrieren zur gesundheitlichen Versorgung zurückgeführt werden.

Soweit es um das individuelle Gesundheitsverhalten geht, hat die Politik inzwischen mit einer Vielzahl von Maßnahmen reagiert - zu einem erheblichen Teil informatorisch-aufklärerischer Art, zum Teil aber auch mit Ge- und Verboten, z.B. im Bereich der Tabakwerbung oder des Nichtraucherschutzes. Die größten Vorbehalte gegen eine derartige „Public Health“-Politik dürften sich aus einem Aspekt ergeben, der im Folgenden näher diskutiert werden soll: Wenn und soweit diese Politik auf die lebensstilbezogenen Gesundheitsfaktoren bezogen ist, scheint sie unweigerlich einen freiheitsfeindlichen und paternalistischen Zug zu erhalten. Sie könnte daher in eine präventionsstaatliche Erziehungs- und Gesundheitsdiktatur des ,healthism“ umschlagen, wenn sie auf die gesundheitsrelevanten Lebensgewohnheiten der Bürger Einfluss zu nehmen sucht. Die Public-Health-Politik ist daher, soweit sie sich auf die Verhaltensprävention konzentriert, dem Einwand ausgesetzt, die Autonomie und Eigenverantwortung der Bürger nicht ernst genug zu nehmen.

\section{Begründungen einer verhaltensbezogenen Public-Health-Politik}

Tatsächlich fragt es sich sehr ernsthaft, welche Legitimation die öffentliche Gewalt besitzt, um auf die gesundheitsrelevante Lebensführung der Bürger einzuwirken. In einer freiheitlichen Ordnung und unter dem Gebot der staatlichen Neutralität mit Bezug auf unterschiedliche Lebensformen kann diese Frage wiederum nur mit Blick auf die möglichen Begründungen dieser Maßnahmen geklärt werden.

Am nächsten liegt das Argument, dass das Gemeinwesen ein Interesse an einer gesundheitsbewussten Lebensführung hat, weil dadurch Kosten reduziert werden. ${ }^{44}$ Dies ist ein zunächst unverdächtiger Ansatz, weil er auf dem bekann-

44 Vgl. etwa Erik Händeler, Die echte Gesundheitsreform, Die BKK 2008, S. 382 ff. 
ten Grundsatz beruht, dass externe Kosten zu internalisieren sind ${ }^{45}$ Soweit damit die Kosten der Sozialsysteme und insbesondere der medizinischen Versorgung gemeint sind, ist aber zum einen nicht sehr klar, ob und unter welchen Umständen dies zutreffend ist. Bekanntlich könnte es sein, dass etwa das Rauchen für die sozialen Systeme letztlich kostenneutral ist. Zum anderen scheint die Beeinflussung der Lebensstile der falsche Ansatzpunkt zu sein; konsequent wäre es, die Betroffenen unmittelbar an den Behandlungskosten ihrer Krankheiten zu beteiligen. Soweit man in die zu berücksichtigenden Kosten darüber hinaus auch den Arbeitsausfall und sonstige gesellschaftliche Folgekosten einbezieht, wird man berücksichtigen müssen, dass dieses Vorgehen einer freiheitlichen Gesellschaftsordnung eher fremd ist: Denn auch unabhängig vom Gesundheitsverhalten haben die Bürger hier keine Pflicht, sich zu möglichst produktiven Mitgliedern des Gemeinwesens zu entwickeln. Wer etwa seine Talente verkümmern lässt, anstatt sie auszubilden und für das Gemeinwesen einzusetzen, wird zwar typischerweise ein geringeres Markteinkommen erzielen, er wird aber nicht zusätzlich mit einer Strafabgabe belegt.

Ein zweites Argument, das grundsätzlich unverdächtig ist, aber ebenfalls über eine sehr begrenzte Reichweite verfügt, stellt darauf ab, dass ein riskantes Verhalten nicht nur die Gesundheit des Betroffenen, sondern auch das Wohlergehen Dritter gefährden kann. Auf diesem Wege sind insbesondere die gesetzlichen Beschränkungen des Tabakkonsums in der Öffentlichkeit gerechtfertigt worden. Die dazu ergangene Rechtsprechung zeigt allerdings, dass der Schutz der Nichtraucher ausnahmslose Rauchverbote schwerlich trägt: Sind Nichtraucher gar nicht, nur marginal oder mit ihrem Einverständnis betroffen, gerät dieses Argument schnell an seine Grenzen. Soweit das Bundesverfassungsgericht trotzdem strikte Rauchverbote für zulässig hielt, ${ }^{46}$ musste es sich auch bereits heftige Kritik gefallen lassen. ${ }^{47}$ Selbst innerhalb des Gerichts war umstritten, ob damit nicht ,,ein Weg edukatorischer Bevormundung vorgezeichnet [wird], der sich auf weitere Bereiche ausdehnen könnte und dann erstickend wirkt" ${ }^{4}{ }^{48} \mathrm{Zu}-$ dem gibt es zahlreiche gesundheitsgefährdende Verhaltensweisen, die auf Dritte gar keine Auswirkungen haben: Wer sich falsch ernährt oder zu wenig bewegt, schädigt in keinem relevanten Sinne das Wohlergehen Dritter.

Die einschlägigen politischen Maßnahmen tragend, aber in seinem normativen Status heikel ist ein drittes Argument, nach dem es darum geht, die Gesundheit der Bevölkerung insgesamt zu schützen und zu verbessern. Dieser Hinweis

45 Vgl. Eberhard Eichenhofer, Wahl des Lebensstils - Auswirkungen in der sozialen Sicherheit, Die Sozialgerichtsbarkeit 50 (2003), S. 705 ff.

46 BVerfGE 121, S. 317 ff.

47 Vgl. nur Rolf Gröschner, Vom Ersatzgesetzgeber zum Ersatzerzieher, ZG 2008, S. 400 ff.

48 BVerfGE 121, S. 317, 388 - Sondervotum Masing. 
auf die „Volksgesundheit" hat in Deutschland aus historischen Gründen schon begrifflich einen besonders schweren Stand..$^{49}$ Aber auch unabhängig davon stellt sich tatsächlich die Frage, ob es ein derartiges Rechtsgut in einer freiheitlichen Ordnung geben kann. Soweit es um die Folgekosten von Krankheiten oder eine Drittschädigung - etwa bei übertragbaren Krankheiten - geht, lässt sich dieser Ansatz auf die beiden bereits erwähnten Argumente reduzieren. Darüber hinaus scheint Gesundheit aber tatsächlich ein privates Gut zu sein, so dass sich die „Volksgesundheit“ nur als summative Bezeichnung für die individuellen Gesundheitszustände auffassen lässt, die keinen darüber hinausgehenden normativen Gehalt besitzt: Der Einzelne ist nicht Bestandteil eines „Volkskörpers“, für dessen Gesundheitszustand er verantwortlich wäre.

Damit bleibt nur eine vierte Begründung, dass es nämlich um das gesundheitliche Wohlergehen jedes einzelnen Bürgers gehe. Damit betritt die öffentliche Gewalt aber vermintes Gelände: Die Bürger in ihrem eigenen Interesse zu einer gesundheitsbewussteren Lebensführung zu erziehen, kollidiert mit dem Grundsatz, dass der freiheitliche Staat zur Neutralität gegenüber unterschiedlichen Lebensformen und -entscheidungen gezwungen ist. Er darf regulierend eingreifen, soweit die Interessen Dritter oder der Allgemeinheit betroffen sind; eine darüber hinausgehende Bewertungskompetenz besitzt er aber nach verbreiteter Ansicht nicht. Eine perfektionistische oder paternalistische Politik ist ihm daher grundsätzlich versagt. Wie tief dieser Grundsatz auch in die verfassungsrechtliche Bewertung eingedrungen ist, wird dadurch belegt, dass sich das Bundesverfassungsgericht veranlasst gesehen hat, zur Rechtfertigung der gesetzlichen Rauchverbote in Gaststätten zu betonen:

„Die Raucher werden hierbei nicht in unzulässiger Weise bevormundet, ihnen wird
insbesondere kein Schutz vor Selbstgefährdung aufgedrängt. Die Landesnichtraucher-
schutzgesetze zielen weder auf Suchtprävention noch auf den Schutz des Einzelnen vor
sich selbst. Ihr Ziel ist vielmehr der Schutz vor den Gefahren des Passivrauchens. Es geht
um den Schutz der Gesundheit nicht des Rauchers, sondern der Gesundheit der anderen
Personen, die in der jeweiligen Situation nicht selbst rauchen."50

Dass diese Konstruktion des Bundesverfassungsgerichts zutreffend ist, kann man aus zwei Gründen bezweifeln. Zum einen ist nicht klar, wie der Nichtraucherschutz derartig weitgehende und flächendeckende Verbote soll rechtfertigen können, die das Gericht für zulässig hält. Ginge es tatsächlich nur um den „Schutz der Gesundheit der anderen Personen, die in der jeweiligen Situation nicht selbst rauchen“, wäre es sehr viel naheliegender, ihnen aufzugeben, die Orte, an denen geraucht wird, zu meiden. Zum anderen fügen sich die Nicht-

49 Vgl. nur Eike Michael Frenzel, Die Volksgesundheit in der Grundrechtsdogmatik, DÖV 2007, S. 243 ff.

50 BVerfGE 121, S. 317, 359. 
raucherschutzgesetze in ein Ensemble von Maßnahmen ein, die das Rauchen als solches bekämpfen; es ist daher mehr als unwahrscheinlich, dass diesen Gesetzen jeder präventionspolitische Impetus fehlt. Das Verbot einer paternalistischen Erziehungspolitik scheint daher entweder der staatlichen Gesundheitspolitik die Hände zu binden oder zur Heuchelei zu zwingen.

\section{Die Public-Health-Perspektive}

Für die Apologeten einer Public-Health-Politik ist dieses Ergebnis insbesondere mit Blick auf die sozialen Gesundheitsungleichheiten nicht zu akzeptieren. ${ }^{51}$ Zwar betonen sie, dass der soziale Gesundheitsgradient nur zum Teil den Lebensstilen in den verschiedenen sozialen Schichten zugeschrieben werden könne. Trotzdem verlöre die Politik ein Handlungsfeld von erheblicher Bedeutung, müsste sie lebensführungsbezogene Gesundheitsfaktoren und -ungleichheiten ignorieren. An dieser Stelle maßgeblich auf das Prinzip der Eigenverantwortung zu setzen, liefe Gefahr, die Schuld den schon Benachteiligten zuzuweisen (,victim blaming") und die öffentliche Gewalt aus ihrer Verantwortung dafür zu entlassen, die für gesundheitsbewusste Entscheidungen hilfreichen sozialen Strukturen zu fördern. Zudem zeigen die bisherigen Erfahrungen, dass Gewohnheiten der Lebensführung jedenfalls dann außerordentlich änderungsresistent sind, wenn sie in Form von Ermahnungen und Aufklärungskampagnen isoliert angegangen werden; der bloße Appell an die Selbstverantwortung der Bürger führt nicht sehr weit, wenn sich nicht auch die sozialen Rahmenbedingungen ändern.

Diese Aspekte weisen darauf hin - und hier liegt der in normativer Hinsicht entscheidende Punkt -, dass individuelle Verhaltensweisen unterschiedlich stark von der kulturellen und sozialen Umwelt geprägt sein können. Lebensstile wie das Ernährungs- und Bewegungsverhalten werden bereits in der Kindheit angelegt, durch soziale und mediale Einflüsse verstärkt und weisen nicht selten Suchtcharakter auf. Dies sind die typischen Elemente, die uns zur Zurückhaltung mahnen sollten, die Verantwortung für diese Verhaltensweisen und ihre Folgen allein dem Individuum zuzuschreiben; es gibt hier offensichtlich „Ursachen der Ursachen“. Dass das jeweilige Verhalten nicht so frei ist, wie gelegentlich behauptet wird, erkennt man auch unschwer daran, dass viele Bürger ihre gesundheitsschädlichen Angewohnheiten ja selbst ändern wollen, es ihnen aber nicht gelingt. In dieser Situation kann eine Politik, die es ihnen erleichtert,

51 Zur Diskussion vgl. nur Daniel Callahan (Hrsg.), Promoting Healthy Behavior: How Much Freedom? Whose Responsibility?, 2000; Daniel Wikler, Personal and Social Responsibility for Health, in: Sudhir Anand/Fabienne Peter/Amartya Sen (Hrsg.) Public Health, Ethics, and Equity, 2005, S. 109 ff. 
ihren eigentlichen Willen handlungswirksam werden zu lassen, ${ }^{52}$ geradezu als Freiheitsförderung betrachtet werden. ${ }^{53}$

Hier kommt eine grundsätzliche Spannung zwischen den Perspektiven des Public-Health-Ansatzes als einer empirischen Sozialwissenschaft einerseits und des Rechts und der Sozialphilosophie andererseits zum Ausdruck. Während Recht und Gerechtigkeitstheorie von der individuellen Autonomie und ihrer Legitimationskraft ausgehen, ist einem Public-Health-Ansatz diese Perspektive aus prinzipiellen Gründen fremd: Er schaut nicht auf das Individuum und die Freiheit seiner Willensbetätigung, sondern abstrahiert davon und stellt auf einer rein statistischen Ebene ein schichtenspezifisches Gesundheitsverhalten fest, obwohl die konkreten Mitglieder dieser Schicht wechseln. Dies legt den Gedanken nahe, dass nicht die Individuen selbst, sondern die sozialen Einflussfaktoren für diese Regelmäßigkeiten und die Unterschiede zwischen den sozialen Einheiten verantwortlich sind. Nun muss diese Spannung zwischen einer Teilnehmerperspektive, in der wir uns selbst als freie und verantwortliche Akteure wahrnehmen und auch anderen diese Eigenschaft zuschreiben, und einer Beobachterperspektive, die den sozialen und kulturellen Hintergrund unserer Entscheidungen und unseres Verhaltens betont, nicht in einem unauflöslichen Gegensatz enden. Vielmehr sind wir es auch in anderen Lebensbereichen gewohnt, die individuellen Entscheidungen zu respektieren, ohne deshalb behaupten zu müssen, dass Individuen unabhängig von diesen Hintergrundfaktoren existieren. Wie die Verantwortung zwischen Individuum und Gesellschaft aufgeteilt wird, muss dann allerdings das Gemeinwesen für jeden Sachbereich immer wieder neu aushandeln und festlegen. ${ }^{54}$

\section{Juristische Konsequenzen}

Vor diesem Hintergrund müssen Beobachter- und Teilnehmerperspektive auch im Recht zu einem Ausgleich gebracht werden. Dies dürfte zum einen bedeuten, dass das Recht die individuelle Entscheidungsfreiheit vor einem gesundheitspolitischen Aktivismus schützen muss. Aus der Anerkennung der Wirkungsmächtigkeit sozialer Faktoren folgt nicht, dass die individuellen Entscheidungen nicht respektiert werden müssten. Dass die sozialontologische These von der sozialen und kulturellen Bedingtheit individuellen Verhaltens richtig ist, gibt dem Ge-

52 Vgl. dazu grundlegend Harry G. Frankfurt, Freedom of the Will and the Concept of a Person, The Journal of Philosophy 68 (1971), S. $5 \mathrm{ff}$.

53 Vgl. M. Gregg Bloche, Obesity and the Struggle Within Ourselves, The Georgetown LJ 93 (2005), S. 1335 ff.

54 John E. Roemer, A Pragmatic Theory of Responsibility for the Egalitarian Planners, Philosophy \& Public Affairs 22 (1993), S. 146 ff. 
meinwesen den Auftrag, diese Bedingungen zu pflegen; es gibt ihm aber in normativer Hinsicht nicht das Recht, dieses Verhalten zu unterbinden. Diese Differenzierung ist aus der sozialphilosophischen Diskussion zwischen liberalen und kommunitaristischen Theorien bekannt. ${ }^{55}$ Angesichts des Umstandes, dass es hier um höchstpersönliche Aspekte der Lebensgestaltung geht, ist eine Präventions- und Gesundheitsförderungspolitik in einer freiheitlichen Ordnung weithin nur als Information, Beratung, Ermunterung und gesundheitsdienliche Ausgestaltung der Lebenswelten, typischerweise dagegen nicht in Form von rechtsverbindlichen Ge- und Verboten möglich: Wer sich bei hinreichender Information und Willensfreiheit für einen ungesunden Lebensstil entscheidet, muss sich in einem freiheitlichen Gemeinwesen nicht daran hindern lassen.

Zum anderen wird die Rechtsordnung aber auch anerkennen müssen, dass die Zuschreibung individueller Verantwortung nicht ohne Berücksichtigung der empirischen Zusammenhänge erfolgen muss und kann. Vermutlich wird man hier zu einer Abstufung der Art kommen, dass politische Interventionen umso eher möglich sind, je stärker der Verdacht ist, dass sich in einem gesundheitsriskanten Verhalten nicht das wohlverstandene Interesse des Individuums, sondern die sozialen Hintergrundfaktoren Bahn brechen.

\section{Fazit}

Die Diskussion der beiden unterschiedlichen Problemfelder - Schulerziehung und Gesundheitsaufklärung - legt die Folgerung nahe, dass Extrempositionen schwer vertretbar sind: Weder kommt dem Staat ein umfassendes Erziehungsmandat $\mathrm{zu}$, noch ist ihm jede erzieherische Einwirkung schlechthin verboten. Es bleibt daher nichts anderes übrig, als die jeweilige Fallkonstellation dogmatisch kleinzuarbeiten, indem die Grundrechte und die aus ihnen abgeleiteten Prinzipien der Verhältnismäßigkeit und der staatlichen Neutralität in Anschlag gebracht werden. Diese Prüfung setzt insbesondere voraus, dass man sich zuvor über die Begründungen des staatlichen Handelns Rechenschaft ablegt.

55 Vgl. dazu etwa Rainer Forst, Kontexte der Gerechtigkeit, 1994. 
